\title{
Slowly varying modes in a two-dimensional duct with shear flow and lined walls
}

\author{
S. W. Rienstra $\dagger$ \\ Department of Mathematics and Computer Science, P.O. Box 513, 5600 MB Eindhoven, The Netherlands
}

(Received 17 January 2020; revised 7 June 2020; accepted 10 August 2020)

A slowly varying modes solution of Wentzel-Kramers-Brillouin type is derived for the problem of sound propagation in a slowly varying two-dimensional duct with homentropic inviscid sheared mean flow and acoustically lined walls of slowly varying impedance. The modal shape function and axial wavenumber are described by the Pridmore-Brown eigenvalue equation. The slowly varying modal amplitude is determined in the usual way by an equation resulting from a solvability condition. For a general mean flow, this equation can be solved in the form of an incomplete adiabatic invariant. Due to conservation of specific mean vorticity along streamlines, two simplifications prove possible for a linearly sheared mean flow: (i) an analytically exact approximation for the mean flow, and (ii) a complete adiabatic invariant for the acoustics. For this last configuration some example cases are evaluated numerically, where the Pridmore-Brown eigenvalue problem is solved by a Galerkin projection combined with an efficient nonlinear iteration.

Key words: aeroacoustics, computational methods

\section{Introduction}

Sound propagation in uniformly lined straight ducts with uniform mean flow is well established by its analytically exact description in duct modes (Rienstra 2003b, 2016a; Rienstra \& Hirschberg 2004). In cross-wise non-uniform flow there are still modes, although the (Pridmore-Brown) equation that describes them is in general not solvable in terms of standard functions (Pridmore-Brown 1958; Vilenski \& Rienstra 2007a,b; Oppeneer et al. 2011; Brambley, Rienstra \& Darau 2012; Oppeneer, Rienstra \& Sijtsma 2016; Rienstra 2020). An exception is two-dimensional (2-D) linear shear flow and uniform sound speed, for which it can be solved exactly by Weber's parabolic cylinder functions (Goldstein \& Rice 1973).

These modal solutions provide insight, but the important effects due to the variation of the duct geometry and the corresponding mean flow cannot be described exactly. At least in slowly varying ducts a systematic approximate solution is possible. The inherently smooth variation of a flow duct provides a small parameter $\varepsilon$ (the slenderness of the duct wall variation) that allows a multiple-scale approach for mean flow and slowly varying

$\dagger$ Email address for correspondence: s.w.rienstra@tue.nl 
modes of WKB (Wentzel-Kramers-Brillouin) type (Nayfeh \& Telionis 1973; Nayfeh, Kaiser \& Telionis 1975; Rienstra 1999, 2000, 2003a; Cooper \& Peake 2001; Peake \& Cooper 2001; Ovenden 2002, 2005; Ovenden \& Rienstra 2004; Ovenden, Eversman \& Rienstra 2004; Brambley \& Peake 2008; Lloyd \& Peake 2013). In the WKB approach it is assumed that the wave retains its shape. If the medium changes slowly enough (meaning little change over one wavelength), the modal order, i.e. the index of the solution of the dispersion relation that defines the mode, remains the same, and the mode does not jump to another eigenstate. The wave function may gradually stretch or compress but the number of nodes and anti-nodes will not change. Under suitable conditions this may be represented mathematically by conserved quantities called adiabatic invariants (Garrett 1967). They may be related to conserved energy, but not necessarily. If an adiabatic invariant can be found (this may be far from trivial, or not possible at all) the WKB solution is almost as transparent as its straight duct counterpart.

The procedure for slowly varying modes in mean flow is, broadly speaking, as follows. First the mean flow is assumed (Bouthier 1972) to be slowly varying everywhere (no entrance effects) so the mean flow equations are rewritten in the slow coordinate $X=\varepsilon x$, rescaled and simplified to leading order in $\varepsilon$, the small parameter that measures the slenderness of the duct variations. These equations are solved analytically if possible (apart from an algebraic equation, this is usually the case for potential flow: Rienstra 1999; Peake \& Cooper 2001; Rienstra 2003a; Brambley \& Peake 2008), or otherwise numerically (often for rotational flow; Cooper \& Peake 2001; Lloyd \& Peake 2013). Then, for the acoustic equations a slowly varying mode is assumed of the form $N(X) \psi(X, y, z) \exp \left(-\mathrm{i} \int^{x} \kappa(\varepsilon z) \mathrm{d} z\right)$, where $\psi$ is conveniently normalised. This particular form, also known as the WKB ansatz, is reasonable because it would emerge from a multiple-scale approach as well. The underlying suppression of secular terms is equivalent to assuming this form. Its existence requires in WKB theory an equation (also known as solvability condition) of the form

$$
\frac{\mathrm{d}}{\mathrm{d} X}\left(N^{2} F\right)=N^{2} G
$$

where $F$ and $G$ are functions of $X$ that depend on cross-sectional integrals of squares and products of $\psi, \psi_{X}, \psi_{y}$ and $\psi_{z}$. For the second-order linear equations of acoustic modes in ducts, this is always possible (cf. Bouthier 1972). If a form can be found without the presence of $\psi_{X}$, the relation between $N$ and $\psi$ is really a local property and we will call this relation an 'incomplete adiabatic invariant', to acknowledge the fact that the solution for $N^{2}$ still includes an integration of $G / F$ to $X$. If also $G=0$, the solvability condition is completely integrable and we will call the resulting integral $N^{2} F=$ constant a 'complete adiabatic invariant', or just 'adiabatic invariant'. In that case the scaling of $\psi$ may be the invariant itself. Note that we do not follow Cooper \& Peake (2001) who called the full equation (1.1), without restrictions on the occurrence of $\psi_{X}$, an adiabatic invariant.

The first solutions of the present type were given by Nayfeh and co-workers (modes in annular flow ducts; Nayfeh et al. 1975). For a number of reasons no adiabatic invariant was found. Firstly, integration of the solvability condition is unlikely because their mean flow was an ad hoc model that does not systematically approximate the equations. Secondly, to include a boundary layer they adopted a sheared flow for which as yet no adiabatic invariants are known.

This is different for potential mean flows. For the solutions with irrotational flow (Rienstra 1999; Peake \& Cooper 2001; Rienstra 2003a; Brambley \& Peake 2008), generally a complete adiabatic invariant exists. 
Following these, great steps were taken by Peake et al. for (systematic) rotational mean flow in cylindrical ducts, with swirl (Cooper \& Peake 2001) and without swirl (Lloyd \& Peake 2013). The complexity of the three-dimensional (3-D) geometry and mean flows, however, prevents the finding (and possibly existence) of both a complete or even incomplete adiabatic invariant and an analytically exact mean flow.

In the present paper we aim to extend Peake and colleagues' theory for slowly varying modes in non-uniform shear flow. We will show that it is possible to make progress by considering a 2-D duct. For a general mean flow an incomplete adiabatic invariant can be found, while for the particular case of a linearly sheared profile the mean flow equations can be solved analytically exactly and for the acoustics a complete adiabatic invariant exists.

The present work builds on, but at the same time improves and replaces, a previous WKB analysis of the related problem with hard walls, published as a conference paper (Rienstra 2016b). Apart from some minor notational differences (like $\Omega$ for $\Omega / C, \Psi$ for $M, \kappa$ for $\mu$ ) and algebraic corrections, the main differences are the slowly varying lined walls, the found complete and incomplete adiabatic invariants and the use of a numerical solution of the central Pridmore-Brown equation, giving access to a very wide range of parameters.

\section{The problem}

\subsection{The equations}

In the acoustic, i.e. isentropic realm of a perfect gas that we will consider, we have for pressure $\tilde{p}$, velocity $\tilde{\boldsymbol{v}}$, density $\tilde{\rho}$, entropy $\tilde{s}$ and sound speed $\tilde{c}$ :

$$
\left.\begin{array}{rl}
\frac{\mathrm{d} \tilde{\rho}}{\mathrm{d} t}=-\tilde{\rho} \nabla \cdot \tilde{\boldsymbol{v}}, \quad \tilde{\rho} \frac{\mathrm{d} \tilde{\boldsymbol{v}}}{\mathrm{d} t}=-\nabla \tilde{p}, \\
\frac{\mathrm{d} \tilde{p}}{\mathrm{~d} t}-\frac{C_{P}}{\tilde{\rho}} \frac{\mathrm{d} \tilde{\rho}}{\mathrm{d} t}=0, \quad \tilde{c}^{2}=\frac{\gamma \tilde{p}}{\tilde{\rho}}, \quad \gamma=\frac{C_{P}}{C_{V}},
\end{array}\right\}
$$

where $\gamma, C_{P}$ and $C_{V}$ are gas constants and $\mathrm{d} / \mathrm{d} t=\partial_{t}+\boldsymbol{v} \cdot \nabla$ denotes the convective derivative; $C_{V}$ is the heat capacity at constant volume, $C_{P}$ is the heat capacity at constant pressure and $\gamma=C_{P} / C_{V}$. Under isentropic conditions, entropy $\tilde{s}$ is constant along a streamline. We will assume a bit more, namely, the constant $\tilde{s}=s_{\text {ref }}$ is the same for all streamlines, which is homentropic flow. In that case we can integrate the entropy equation to get

$$
C_{V} \log \tilde{p}-C_{P} \log \tilde{\rho}=s_{r e f}=C_{V} \log p_{r e f}-C_{P} \log \rho_{r e f}
$$

or

$$
\frac{\tilde{p}}{p_{\text {ref }}}=\left(\frac{\tilde{\rho}}{\rho_{\text {ref }}}\right)^{\gamma} .
$$

When we have a stationary mean flow with unsteady time-harmonic perturbations of frequency $\omega$, given, in the usual complex notation, by

$$
\tilde{\boldsymbol{v}}=V+\operatorname{Re}\left(\boldsymbol{v} \mathrm{e}^{\mathrm{i} \omega t}\right), \quad \tilde{p}=P+\operatorname{Re}\left(p \mathrm{e}^{\mathrm{i} \omega t}\right), \quad \tilde{\rho}=D+\operatorname{Re}\left(\rho \mathrm{e}^{\mathrm{i} \omega t}\right),
$$


and linearise for small amplitude, we obtain for the mean flow

$$
\left.\begin{array}{rl}
\nabla \cdot(D V) & =0, \quad D(V \cdot \nabla) V=-\nabla P \\
\frac{P}{p_{r e f}} & =\left(\frac{D}{\rho_{r e f}}\right)^{\gamma}, \quad C^{2}=\frac{\gamma P}{D} .
\end{array}\right\}
$$

The mean flow momentum equation can be integrated along streamlines to yield (Batchelor 1967) a version of Bernoulli's equation that the total specific enthalpy

$$
\frac{1}{2}|V|^{2}+\frac{C^{2}}{\gamma-1}=\text { constant along a streamline, }
$$

not necessarily the same constant for all streamlines. Another quantity, conserved along streamlines, that will be of interest is the specific vorticity $\Xi / D$, where $\Xi=V_{x}-U_{y}$. Take the curl of the mean flow momentum equation and substitute the mass conservation to get, by virtue of the pressure being barotropic (Batchelor 1967),

$$
V \cdot \nabla\left(\frac{\Xi}{D}\right)=0 .
$$

Finally, the perturbations are described by the equations

$$
\left.\begin{array}{c}
\mathrm{i} \omega \rho+\nabla \cdot(\boldsymbol{V} \rho+\boldsymbol{v} D)=0, \\
D(\mathrm{i} \omega+\boldsymbol{V} \cdot \boldsymbol{\nabla}) \boldsymbol{v}+D(\boldsymbol{v} \cdot \boldsymbol{\nabla}) \boldsymbol{V}+\rho(\boldsymbol{V} \cdot \boldsymbol{\nabla}) \boldsymbol{V}=-\nabla p, \\
p=C^{2} \rho .
\end{array}\right\}
$$

Myer's time-averaged acoustic energy equations for inviscid and homentropic flow, with $\Xi$ and $\xi$ the mean and perturbed flow vorticity, are given by (Myers 1991; Rienstra \& Hirschberg 2004)

$$
\begin{gathered}
\nabla\langle\boldsymbol{I}\rangle=-\langle\mathcal{D}\rangle, \\
\langle\boldsymbol{I}\rangle=\frac{1}{2} \operatorname{Re}\left[(D \boldsymbol{v}+\rho \boldsymbol{V})\left(\frac{p^{*}}{D}+\boldsymbol{V} \cdot \boldsymbol{v}^{*}\right)\right], \\
\langle\mathcal{D}\rangle=-\frac{1}{2} \operatorname{Re}\left[\xi D \boldsymbol{V} \cdot\left(-v^{*}, u^{*}\right)+\Xi \rho \boldsymbol{v}^{*} \cdot(-V, U)\right] .
\end{gathered}
$$

\subsection{Non-dimensionalisation}

Without further change of notation, we will assume throughout this paper that the problem is made dimensionless: lengths on a typical duct height $a_{r e f}$, velocities on a typical sound speed $c_{r e f}$, time on $a_{r e f} / c_{r e f}$, densities on a typical density $\rho_{\text {ref }}$ and pressures on $\gamma p_{r e f}=$ $\rho_{\text {ref }} c_{r e f}^{2}$. The dimensionless equations are the same as above, except

$$
\gamma P=D C^{2}=D^{\gamma}
$$

while dimensionless frequency $\omega$ is also known as the Helmholtz number. 


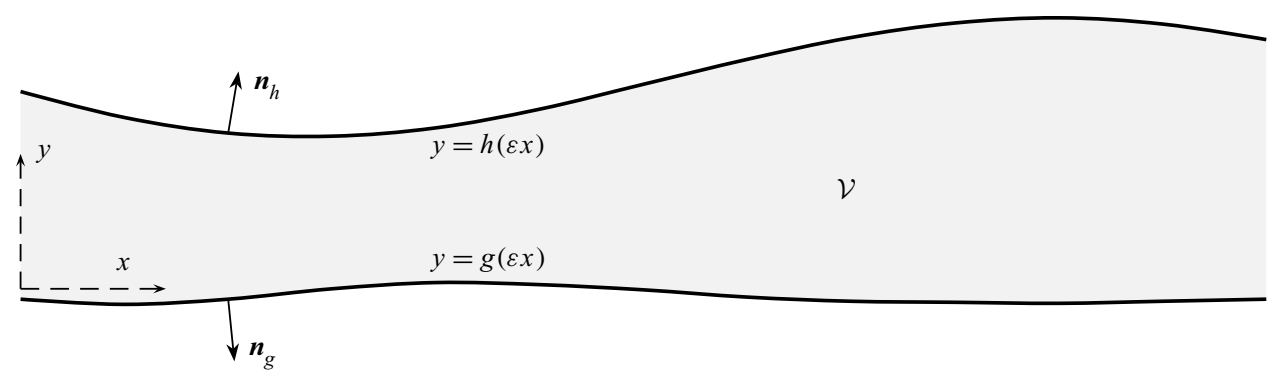

FIGURE 1. Sketch of geometry: two-dimensional slowly varying duct.

\subsection{The geometry}

The domain of interest, sketched in figure 1, is a 2-D duct $\mathcal{V}$ of slowly varying cross-section $g(X) \leq y \leq h(X)$, where $0<h-g=O(1) ; X=\varepsilon x$ is a so-called slow variable while $\varepsilon$ is small. At the duct top surface $y=h$ and the bottom surface $y=g$, the gradients

$$
\nabla(y-h)=\boldsymbol{e}_{y}-\varepsilon \boldsymbol{e}_{x} h_{X}, \quad \nabla(y-g)=\boldsymbol{e}_{y}-\varepsilon \boldsymbol{e}_{x} g_{X},
$$

are vectors normal to the surface, so the outward normals of the top and bottom surface are given by

$$
\left.\begin{array}{c}
\boldsymbol{n}_{h}=\frac{\boldsymbol{e}_{y}-\varepsilon \boldsymbol{e}_{x} h_{X}}{\sqrt{1+\varepsilon^{2} h_{X}^{2}}}=\boldsymbol{e}_{y}-\varepsilon \boldsymbol{e}_{x} h_{X}+O\left(\varepsilon^{2}\right), \\
\boldsymbol{n}_{g}=-\frac{\boldsymbol{e}_{y}-\varepsilon \boldsymbol{e}_{x} g_{X}}{\sqrt{1+\varepsilon^{2} g_{X}^{2}}}=-\boldsymbol{e}_{y}+\varepsilon \boldsymbol{e}_{x} g_{X}+O\left(\varepsilon^{2}\right) .
\end{array}\right\}
$$

Evidently, $\boldsymbol{e}_{x}$ and $\boldsymbol{e}_{y}$ denote the unit vectors in the $x$ and $y$ directions, the indices $h$ and $g$ refer to the top and bottom walls and the index $X$ to a partial derivative to $X$. Except with $\boldsymbol{e}_{y}$, the index $y$ will be used to denote a partial derivative to $y$.

\subsection{Boundary conditions}

If we denote the mean flow by $V=U \boldsymbol{e}_{x}+V \boldsymbol{e}_{y}$, the impermeable duct wall yields the mean flow boundary condition $(\boldsymbol{V} \cdot \boldsymbol{n})=0$, or

$$
V-\varepsilon g_{X} U=0 \quad \text { at } y=g
$$

and

$$
V-\varepsilon h_{X} U=0 \quad \text { at } y=h .
$$

The mean flow mass flux, given by

$$
\int_{g}^{h} D U \mathrm{~d} y=\mathcal{F},
$$

is then independent of $x$, where $\mathcal{F}$ is (after non-dimensionalisation) a constant $O(1)$. The mean flow is assumed to be determined by the slowly varying geometry only.

The acoustic boundary condition of the slowly varying lined wall includes the refraction through the vanishing boundary layer, and is a form of the so-called Ingard-Myers 
condition (Ingard 1959; Myers 1980), given at $y=h(i=h)$ and $y=g(i=g)$ by

$$
\boldsymbol{v} \cdot \boldsymbol{n}_{i}=\left(\mathrm{i} \omega+\boldsymbol{V} \cdot \nabla-\boldsymbol{n}_{i} \cdot\left(\boldsymbol{n}_{i} \cdot \nabla V\right)\right)\left(\frac{p}{\mathrm{i} \omega Z_{i}}\right) .
$$

The impedances may vary slowly with position, so we can write $Z_{i}=Z_{i}(X)$. It is important to note that $Z_{i}$ is only defined along a wall, and only derivatives along wall streamlines have meaning.

\section{Mean flow}

\subsection{The general case}

Since we assumed the mean flow to be determined by the slowly varying geometry only, we write all flow variables as a function of $(X, y ; \varepsilon)$, and expand each in a regular Poincare expansion in powers of $\varepsilon^{2}$ (the small parameter that appears in the equations). From elementary order of magnitude considerations it follows that $U=O(1), V=O(\varepsilon)$, $D=O(1), C=O(1)$ and $P=O(1)$. So we have

$$
\left.\begin{array}{c}
U=U_{0}+O\left(\varepsilon^{2}\right), \quad V=\varepsilon V_{0}+O\left(\varepsilon^{3}\right), \\
D=D_{0}+O\left(\varepsilon^{2}\right), \quad P=P_{0}+O\left(\varepsilon^{2}\right), \quad C=C_{0}+O\left(\varepsilon^{2}\right),
\end{array}\right\}
$$

where each term in the expansion is independent of $\varepsilon$. For notational convenience we leave out the 0 subscript, because higher orders will not be considered.

We substitute these expansions in the conservation equations and collect the terms of like powers of $\varepsilon$. Then we get to leading order

$$
\left.\begin{array}{c}
(D U)_{X}+(D V)_{y}=0 \\
D\left(U U_{X}+V U_{y}\right)+P_{X}=0 \\
P_{y}=0
\end{array}\right\}
$$

with boundary conditions

$$
V-g_{X} U=0 \quad \text { at } y=g \quad \text { and } \quad V-h_{X} U=0 \quad \text { at } y=h .
$$

From $P=P(X)$ is also $D=D(X)$ and $C=C(X)$, and so $P_{X}=C^{2} D_{X}=D^{\gamma-1} D_{X}$. From the flux condition (2.14) we find

$$
D(X)=\frac{\mathcal{F}}{\int_{g}^{h} U(X, y) \mathrm{d} y} .
$$

Furthermore, the mass and momentum equations become

$$
\left.\begin{array}{c}
(D U)_{X}+D V_{y}=0, \\
D U U_{X}+D V U_{y}+D^{\gamma-1} D_{X}=0,
\end{array}\right\}
$$

of which the last equation can be written as

$$
U \frac{\partial}{\partial X}\left(\frac{1}{2} U^{2}+\frac{D^{\gamma-1}}{\gamma-1}\right)+V \frac{\partial}{\partial y}\left(\frac{1}{2} U^{2}+\frac{D^{\gamma-1}}{\gamma-1}\right)=0 .
$$


This again amounts to the approximated Bernoulli's equation (2.6), saying that

$$
\frac{1}{2} U^{2}+\frac{D^{\gamma-1}}{\gamma-1}=\text { constant along streamlines, }
$$

for example, the wall streamlines at $y=g$ and $y=h$.

By combining the mass equation and $y$-derivative of the momentum equation, we find the approximated version of (2.7)

$$
U \frac{\partial}{\partial X}\left(\frac{U_{y}}{D}\right)+V \frac{\partial}{\partial y}\left(\frac{U_{y}}{D}\right)=0
$$

which means that the specific mean shear

$$
\frac{U_{y}}{D}=\text { constant along streamlines. }
$$

If we start at (say) $X=X_{0}$ with a linearly sheared flow, i.e. $U_{y}=\sigma_{0}$, then the conserved constant $\lambda=\sigma_{0} / D\left(X_{0}\right)$ is the same for all $X$ and $y$, and $U_{y}=\lambda D$ everywhere.

The equation of mass conservation leads to

$$
D V=\left.g_{X} D U\right|_{y=g}-\int_{g}^{y}(D U)_{X} \mathrm{~d} y^{\prime},
$$

which satisfies the boundary condition at $y=g$ (by construction), and at $y=h$ because

$$
D V-h_{X} D U=-\frac{\mathrm{d}}{\mathrm{d} X} \int_{g}^{h} D U \mathrm{~d} y=-\frac{\mathrm{d}}{\mathrm{d} X} \mathcal{F}=0 .
$$

For a potential mean flow the above conditions would be sufficient to define the flow (Rienstra 2003a). For example, $U$ can only be constant in $y$, and the other variables follow by an algebraic equation to be solved at each position $X$. For the vortical mean flow we have here, we need to define an initial velocity profile $(U, \varepsilon V)$ and integrate numerically (iteratively) along streamlines while using (3.3), (3.6) and (3.9). This is not straightforward. See for example Lloyd \& Peake (2013) for the closely related cylindrical version of this problem where use is made of the streamfunction.

\subsection{Linearly sheared flow}

A numerical evaluation of the mean flow is, however, not always necessary. Hinted at by (3.8) and its following paragraph, it appears that for a velocity profile that is linear in $y$, we can find a solution that avoids a numerical integration and much like the potential flow solution requires no more than solving an algebraic equation. Even more, an accidental property will, rather unexpectedly, give our acoustic solution for this case the bonus of a complete adiabatic invariant. 
The type of velocity profile we will consider is to leading order given by

$$
U(X, y)=\tau(X)+\sigma(X)(y-g(X)),
$$

where $\sigma$ and $\tau$ are to be determined from the conditions of fixed mass flux and varying wall normal. Then

$$
D V=g_{X} D U-(D \tau)_{X}(y-g)-\frac{1}{2}(D \sigma)_{X}(y-g)^{2},
$$

and

$$
D \tau \tau_{X}+D^{\gamma-1} D_{X}+\left(D \sigma_{X}-D_{X} \sigma\right)\left(\tau(y-g)+\frac{1}{2} \sigma(y-g)^{2}\right)=0 .
$$

Equation (3.13) is valid for any $X$ and $y$. Its $y$-dependence is only through the polynomial $\tau(y-g)+\frac{1}{2} \sigma(y-g)^{2}$. This vanishes for $y=g$, but is otherwise non-zero (except for the uninteresting case $\tau=\sigma=0$, and possibly a single point $y=g-2 \tau / \sigma)$. Therefore (3.13) requires

$$
\begin{gathered}
D \tau \tau_{X}+D^{\gamma-1} D_{X}=0, \\
D \sigma_{X}-D_{X} \sigma=0,
\end{gathered}
$$

both of which can be integrated straightaway. So $\sigma / D=$ constant, which agrees with (3.8). Altogether, we find that for any physically allowable (e.g. no supersonic pockets) constants $\lambda$ and $E$, a mean flow exists given by

$$
\sigma=\lambda D
$$

and Bernoulli's equation (3.6) along streamline $y=g$ :

$$
\frac{1}{2} \tau^{2}+\frac{1}{\gamma-1} D^{\gamma-1}=E \text {. }
$$

Eliminate $\tau$ from

$$
\int_{g}^{h} D U \mathrm{~d} y=D \int_{g}^{h} U \mathrm{~d} y=D(h-g)\left(\tau+\frac{1}{2} \sigma(h-g)\right)=\mathcal{F}
$$

to get

$$
\tau=\frac{\mathcal{F}}{D(h-g)}-\frac{1}{2} \sigma(h-g),
$$

by which Bernoulli's equation (3.6) applies along streamline $y=h$, with constant $E+$ $\lambda \mathcal{F}$. Altogether, we have for given geometry $h$ and $g$ and mean flow constants $\lambda, E$ and $\mathcal{F}$, the following algebraic equation:

$$
\frac{1}{2}\left(\frac{\mathcal{F}}{D(h-g)}-\frac{1}{2} \lambda D(h-g)\right)^{2}+\frac{1}{\gamma-1} D^{\gamma-1}=E,
$$

to be solved per $X$ for $D$. Then we have $\tau, \sigma, U$ and $C$ like above, from which $V$ follows

$$
V=g_{X} U+\left.U\right|_{y=h} \frac{C^{2}-\tau U}{C^{2}-\left.\tau U\right|_{y=h}} \frac{h_{X}-g_{X}}{h-g}(y-g) .
$$

Note that there is apparently no slowly varying mean flow of linear shear with $\tau(X) \equiv 0$. 


\section{Acoustic field}

\subsection{Slowly varying modes}

With the mean flow variables expanded to leading order, and after eliminating $\rho=p / C^{2}$, we have the acoustic equations

$$
\begin{gathered}
\mathrm{i} \omega p+U p_{x}+D C^{2}\left(u_{x}+v_{y}\right)=-\varepsilon\left[C^{2}\left(\frac{U}{C^{2}}\right)_{X} p+C^{2} D_{X} u+(V p)_{y}\right]+O\left(\varepsilon^{2}\right), \\
D\left(\mathrm{i} \omega u+U u_{x}\right)+D U_{y} v+p_{x}=-\varepsilon\left[-D^{-1} D_{X} p+D\left(U_{X} u+V u_{y}\right)\right]+O\left(\varepsilon^{2}\right), \\
D\left(\mathrm{i} \omega v+U v_{x}\right)+p_{y}=-\varepsilon D(V v)_{y}+O\left(\varepsilon^{2}\right),
\end{gathered}
$$

and the lined wall boundary condition at $y=h(X)$ and $y=g(X)$, respectively,

$$
\left.\begin{array}{c}
v-\varepsilon h_{X} u=\left(\mathrm{i} \omega+U \frac{\partial}{\partial x}+\varepsilon V \frac{\partial}{\partial y}-\varepsilon V_{y}+\varepsilon h_{X} U_{y}\right)\left(\frac{p}{i \omega Z_{h}}\right)+O\left(\varepsilon^{2}\right), \\
-\left(v-\varepsilon g_{X} u\right)=\left(i \omega+U \frac{\partial}{\partial x}+\varepsilon V \frac{\partial}{\partial y}-\varepsilon V_{y}+\varepsilon g_{X} U_{y}\right)\left(\frac{p}{i \omega Z_{g}}\right)+O\left(\varepsilon^{2}\right) .
\end{array}\right\}
$$

We seek slowly varying modes of the form

$$
[p, u, v]=[\Psi(X, y ; \varepsilon), A(X, y ; \varepsilon), B(X, y ; \varepsilon)] \exp \left(-\mathrm{i} \int^{x} \kappa(\varepsilon z ; \varepsilon) \mathrm{d} z\right) \text {. }
$$

Introduce

$$
\Omega=\frac{\omega-\kappa U}{C}, \quad \Omega_{y}=-\frac{\kappa U_{y}}{C},
$$

and substitute (4.2) in (4.1) to obtain after some simplifications to $O\left(\varepsilon^{2}\right)$

$$
\left.\begin{array}{c}
\Omega^{2} \Psi-\kappa D \Omega C A-\mathrm{i} D \Omega C B_{y}=\mathrm{i} \varepsilon \Omega C\left[\left(\frac{U \Psi}{C^{2}}\right)_{X}+\left(\frac{V \Psi}{C^{2}}\right)_{y}+(D A)_{X}\right]+O\left(\varepsilon^{2}\right), \\
\kappa D \Omega C A+\mathrm{i} D \Omega_{y} C B-\kappa^{2} \Psi=\mathrm{i} \varepsilon \kappa D\left[\left(\frac{\Psi}{D}\right)_{X}+(U A)_{X}+V A_{y}\right]+O\left(\varepsilon^{2}\right), \\
\mathrm{i} \frac{D C B}{\Omega}+\frac{\Psi_{y}}{\Omega^{2}}=-\varepsilon \frac{D}{\Omega^{2}}\left[U B_{X}+(V B)_{y}\right]+O\left(\varepsilon^{2}\right),
\end{array}\right\}
$$

and boundary conditions

$$
\left.\begin{array}{c}
B-\varepsilon h_{X} A=\left(\mathrm{i} \Omega C+\varepsilon U \frac{\partial}{\partial X}+\varepsilon V \frac{\partial}{\partial y}-\varepsilon V_{y}+\varepsilon h_{X} U_{y}\right)\left(\frac{\Psi}{\mathrm{i} \omega Z_{h}}\right)+O\left(\varepsilon^{2}\right), \\
-\left(B-\varepsilon g_{X} A\right)=\left(\mathrm{i} \Omega C+\varepsilon U \frac{\partial}{\partial X}+\varepsilon V \frac{\partial}{\partial y}-\varepsilon V_{y}+\varepsilon g_{X} U_{y}\right)\left(\frac{\Psi}{\mathrm{i} \omega Z_{g}}\right)+O\left(\varepsilon^{2}\right) .
\end{array}\right\}
$$


If we take the $y$-derivative of the third equation of $(4.4 a)$, multiply this by $\Omega^{2}$, and add the result to the sum of the first two equations of $(4.4 a)$, we obtain

$$
\begin{gathered}
\Omega^{2}\left(\frac{\Psi_{y}}{\Omega^{2}}\right)_{y}+\left(\Omega^{2}-\kappa^{2}\right) \Psi=\mathrm{i} \varepsilon \Omega^{2}\left[\frac{C}{\Omega}\left(\frac{U \Psi}{C^{2}}+D A\right)_{X}+\frac{\kappa D}{\Omega^{2}}\left(\frac{\Psi}{D}+U A\right)_{X}\right. \\
\left.+\frac{1}{\Omega C}(V \Psi)_{y}+\frac{\kappa D V}{\Omega^{2}} A_{y}+\mathrm{i} D\left(\frac{1}{\Omega^{2}}\left[U B_{X}+(V B)_{y}\right]\right)_{y}\right]+O\left(\varepsilon^{2}\right),
\end{gathered}
$$

while

$$
\left.\begin{array}{c}
A=\frac{\kappa \Psi}{D \Omega C}-\frac{U_{y} \Psi_{y}}{D \Omega^{2} C^{2}}+O(\varepsilon) \\
B=\mathrm{i} \frac{\Psi_{y}}{D \Omega C}-\frac{\varepsilon}{\Omega C}\left[U\left(\frac{\Psi_{y}}{D \Omega C}\right)_{X}+\left(\frac{V \Psi_{y}}{D \Omega C}\right)_{y}\right]+O\left(\varepsilon^{2}\right) .
\end{array}\right\}
$$

We expand to $O\left(\varepsilon^{2}\right)$

$$
A=A_{0}+\varepsilon A_{1}+\ldots, \quad B=B_{0}+\varepsilon B_{1}+\ldots, \quad \Psi=\Psi_{0}+\varepsilon \Psi_{1}+\ldots, \quad \kappa=\kappa_{0}+\ldots
$$

There is no need for a $\kappa_{1}$-term, as $\exp \left(-\mathrm{i} \varepsilon \int^{x} \kappa_{1} \mathrm{~d} z\right)$ depends on $X$ only and can be absorbed by $\Psi, A$ and $B$. For notational convenience we retain $\kappa$ in the formulas. To leading-order, equation (4.5) reduces to the Pridmore-Brown eigenvalue equation

$$
\Omega^{2}\left(\frac{\Psi_{0 y}}{\Omega^{2}}\right)_{y}+\left(\Omega^{2}-\kappa^{2}\right) \Psi_{0}=0,
$$

with boundary conditions

$$
\Psi_{0 y}=\frac{D \Omega^{2} C^{2}}{\mathrm{i} \omega Z_{h}} \Psi_{0}, \quad \Psi_{0 y}=-\frac{D \Omega^{2} C^{2}}{\mathrm{i} \omega Z_{g}} \Psi_{0},
$$

where $\Psi_{0}$ and $\kappa$ are to be determined. Through $g, h, U, C, Z_{g}$ and $Z_{h}, \Psi_{0}$ and $\kappa$ will vary in $X$. Since the equation and boundary conditions are linear and homogeneous, $\Psi_{0}$ is only determined up to a slowly varying amplitude. In the following we will derive an adiabatic invariant for $\Psi_{0}$ to determine this slowly varying factor.

\subsection{Final solution part 1: the general case}

The next order of the expanded equation (4.5) is the inhomogeneous Pridmore-Brown equation in $\Psi_{1}$ :

$$
\begin{gathered}
\Omega^{2}\left(\frac{\Psi_{1 y}}{\Omega^{2}}\right)_{y}+\left(\Omega^{2}-\kappa^{2}\right) \Psi_{1}=\mathrm{i} \Omega^{2}\left[\frac{C}{\Omega}\left(\left(\kappa+\frac{U \Omega}{C}\right) \frac{\Psi_{0}}{\Omega C}-\frac{U_{y} \Psi_{0 y}}{\Omega^{2} C^{2}}\right)_{X}+\frac{\kappa^{2} V \Psi_{0 y}}{\Omega^{3} C}\right. \\
\left.+\frac{\kappa D}{\Omega^{2}}\left(\frac{\omega \Psi_{0}}{D \Omega C}-\frac{U U_{y} \Psi_{0 y}}{D \Omega^{2} C^{2}}\right)_{X}+\left(\frac{V \Psi_{0}}{\Omega C}-\frac{D U}{\Omega^{2}}\left(\frac{\Psi_{0 y}}{D \Omega C}\right)_{X}-\frac{1}{\Omega^{2} C}\left(\frac{V \Psi_{0 y}}{\Omega}\right)_{y}\right)_{y}\right],
\end{gathered}
$$


with boundary conditions (obtained by using the directional derivatives of $(4.8 b)$ along wall streamlines)

$$
\left.\begin{array}{l}
\mathrm{i}\left(\Psi_{1 y}-\frac{D \Omega^{2} C^{2}}{\mathrm{i} \omega Z_{h}} \Psi_{1}\right)=\frac{Z_{h} D U}{\Psi_{0}}\left(\frac{\Psi_{0} \Psi_{0 y}}{Z_{h} D \Omega C}\right)_{X}+\frac{Z_{h} D V}{\Psi_{0}}\left(\frac{\Psi_{0} \Psi_{0 y}}{Z_{h} D \Omega C}\right)_{y}+h_{X} \kappa \Psi_{0}, \\
\mathrm{i}\left(\Psi_{1 y}+\frac{D \Omega^{2} C^{2}}{\mathrm{i} \omega Z_{g}} \Psi_{1}\right)=\frac{Z_{g} D U}{\Psi_{0}}\left(\frac{\Psi_{0} \Psi_{0 y}}{Z_{g} D \Omega C}\right)_{X}+\frac{Z_{g} D V}{\Psi_{0}}\left(\frac{\Psi_{0} \Psi_{0 y}}{Z_{g} D \Omega C}\right)_{y}+g_{X} \kappa \Psi_{0} .
\end{array}\right\}
$$

We do not aim to solve this equation, as it would again involve undetermined homogeneous solutions. Instead, we derive a solvability condition on $\Psi_{0}$, required for $\Psi_{1}$ to exist. For this, we combine equations $(4.8 a)$ and (4.9a) into

$$
\mathrm{i} \int_{g}^{h} \frac{1}{D \Omega^{2} C^{2}}\left([\text { equation }(4.9 a)] \Psi_{0}-[\text { equation }(4.8 a)] \Psi_{1}\right) \mathrm{d} y,
$$

then use the self-adjointness of the Pridmore-Brown equation to eliminate $\Psi_{1}$ and apply the boundary conditions $(4.9 b)$ to obtain

$$
\begin{aligned}
& {\left[\frac{Z U}{\Omega^{2} C^{2}}\left(\frac{\Psi_{0} \Psi_{0 y}}{Z D \Omega C}\right)_{X}+\frac{Z V}{\Omega^{2} C^{2}}\left(\frac{\Psi_{0} \Psi_{0 y}}{Z D \Omega C}\right)_{y}+\frac{V \kappa \Psi_{0}^{2}}{U D \Omega^{2} C^{2}}\right]_{g}^{h}} \\
& =-\int_{g}^{h} \frac{\Psi_{0}}{D C^{2}}\left[\frac{C}{\Omega}\left(\left(\kappa+\frac{U \Omega}{C}\right) \frac{\Psi_{0}}{\Omega C}-\frac{U_{y} \Psi_{0 y}}{\Omega^{2} C^{2}}\right)_{X}+\frac{\kappa^{2} V}{\Omega^{3} C} \Psi_{0 y}\right. \\
& \left.\quad+\frac{\kappa D}{\Omega^{2}}\left(\frac{\omega \Psi_{0}}{D \Omega C}-\frac{U U_{y} \Psi_{0 y}}{D \Omega^{2} C^{2}}\right)_{X}+\left(\frac{V \Psi_{0}}{\Omega C}-\frac{D U}{\Omega^{2}}\left(\frac{\Psi_{0 y}}{D \Omega C}\right)_{X}-\frac{1}{\Omega^{2} C}\left(\frac{V \Psi_{0 y}}{\Omega}\right)_{y}\right)\right] \mathrm{d} y .
\end{aligned}
$$

By several partial integrations, using the defining differential equation and collecting together the $X$-derivative of $(\kappa+U \Omega / C) \Psi_{0}^{2} / D \Omega^{2} C^{2}-\omega U_{y} \Psi_{0} \Psi_{0 y} / D \Omega^{4} C^{4}$, we obtain after a considerable amount of algebra the equation

$$
\begin{aligned}
& {\left[\frac{Z \Psi_{0 y}}{D \Omega^{2} C^{2} \Psi_{0}}\left(\frac{U \Psi_{0}^{2}}{Z \Omega C}\right)_{X}+\frac{Z \Psi_{0 y}}{D \Omega^{2} C^{2} \Psi_{0}} \frac{V}{U}\left(\frac{U \Psi_{0}^{2}}{Z \Omega C}\right)_{y}\right]_{g}^{h}} \\
& \quad+\frac{\mathrm{d}}{\mathrm{d} X} \int_{g}^{h}\left(\kappa+\frac{U \Omega}{C}\right) \frac{\Psi_{0}^{2}}{D \Omega^{2} C^{2}}-\frac{\omega U_{y} \Psi_{0} \Psi_{0 y}}{D \Omega^{4} C^{4}} \mathrm{~d} y=\int_{g}^{h} \frac{\kappa U \Psi_{0} \Psi_{0 y}}{\Omega^{4} C^{4}}\left(\frac{U_{y}}{D}\right)_{X} \mathrm{~d} y .
\end{aligned}
$$

With boundary conditions $(4.8 b)$ this becomes

$$
\begin{gathered}
\frac{\mathrm{d}}{\mathrm{d} X}\left[\left.\frac{1}{\mathrm{i} \omega Z_{h}} \frac{U \Psi_{0}^{2}}{\Omega C}\right|_{h}+\left.\frac{1}{\mathrm{i} \omega Z_{g}} \frac{U \Psi_{0}^{2}}{\Omega C}\right|_{g}+\int_{g}^{h}\left(\kappa+\frac{U \Omega}{C}\right) \frac{\Psi_{0}^{2}}{D \Omega^{2} C^{2}}-\frac{\omega U_{y} \Psi_{0} \Psi_{0 y}}{D \Omega^{4} C^{4}} \mathrm{~d} y\right] \\
=\int_{g}^{h} \frac{\kappa U \Psi_{0} \Psi_{0 y}}{\Omega^{4} C^{4}}\left(\frac{U_{y}}{D}\right)_{X} \mathrm{~d} y .
\end{gathered}
$$

This form is interesting because of its congruence with the acoustic power (see below) in the case of cut-on modes in linearly sheared flow and hard walls. An even more elegant 
form is found by applying the relation (A 1) of appendix A, with $\mathcal{G}=U / D \Omega C^{3}$, and absorbing the boundary terms into the integral, to find

$$
\frac{\mathrm{d}}{\mathrm{d} X}\left[\int_{g}^{h} \frac{1}{D \Omega^{3} C^{3}}\left(\omega \kappa \Psi_{0}^{2}+U \Psi_{0 y}^{2}\right) \mathrm{d} y\right]=\int_{g}^{h} \frac{\kappa U \Psi_{0} \Psi_{0 y}}{\Omega^{4} C^{4}}\left(\frac{U_{y}}{D}\right)_{X} \mathrm{~d} y
$$

If we normalise $\Psi_{0}$ to $\bar{\Psi}_{0}$ in some smooth way and introduce the slowly varying amplitude $N$ such that $\Psi_{0}(X, y)=N(X) \bar{\Psi}_{0}(X, y)$, then the equation for $N$

$$
\frac{\mathrm{d}}{\mathrm{d} X}\left[N^{2} F\right]=N^{2} G,
$$

with

$$
F(X)=\int_{g}^{h} \frac{1}{D \Omega^{3} C^{3}}\left(\omega \kappa \bar{\Psi}_{0}^{2}+U \bar{\Psi}_{0 y}^{2}\right) \mathrm{d} y, \quad G(X)=\int_{g}^{h} \frac{\kappa U \bar{\Psi}_{0} \bar{\Psi}_{0 y}}{\Omega^{4} C^{4}}\left(\frac{U_{y}}{D}\right)_{X} \mathrm{~d} y,
$$

leads to a form of an incomplete adiabatic invariant

$$
N^{2}(X)=N^{2}(0) \frac{F(0)}{F(X)} \exp \left(\int_{0}^{X} \frac{G(z)}{F(z)} \mathrm{d} z\right)
$$

This is similar to Peake \& Cooper (2001), Cooper \& Peake (2001), Lloyd \& Peake (2013) and Rienstra (2016b), although now $F$ and $G$ are independent of $X$-derivatives of $\bar{\Psi}_{0}$.

\subsection{Final solution part 2: linearly sheared flow}

If $\left(U_{y} / D\right)_{X}=0, G(X)=0$ and (4.16) reduces to a complete adiabatic invariant. Since $D$ is independent of $y$, this is the case for a linearly sheared mean flow with the property that $U_{y} / D=$ a constant. As we found above in (3.16), this is not a restriction. It occurs for any linearly sheared flow that is of slow variation! We have then for a constant $Q_{0}$ :

$$
\int_{g}^{h} \frac{1}{D \Omega^{3} C^{3}}\left(\omega \kappa \Psi_{0}^{2}+U \Psi_{0 y}^{2}\right) \mathrm{d} y=Q_{0}
$$

We do not have to normalise $\Psi_{0}$ : the adiabatic invariant is the normalisation. The size of $Q_{0}$ is unimportant in a linear problem, although we can use it to scale the solution conveniently. There is, however, a small reservation. For hard walls we can assume $\Psi$ to be real, in which case the sign of $Q_{0}$ relates to the propagation direction and cannot be chosen freely. This is due to the fact that for hard-wall real cut-on modes this invariant is, apart from a factor $\omega / 2$, equal to the acoustic power passing a duct cross-section $X=$ constant, at least, up to the WKB accuracy. We find for the integral of the time-averaged energy flux 
$\langle I\rangle$ in the $x$-direction (2.9b) (with hard walls, $U_{y y}=0$, real $\Psi$ and ignoring subscript 0 )

$$
\begin{aligned}
\mathcal{P}(X) & =\int_{g}^{h} \frac{1}{2} \operatorname{Re}\left[\left(D A+\frac{U \Psi}{C^{2}}\right)\left(\frac{\Psi}{D}+U A\right)^{*}\right] \mathrm{d} y+O(\varepsilon) \\
& =\frac{1}{2} \int_{g}^{h}\left(\frac{\kappa \Psi}{\Omega C}-\frac{U_{y} \Psi_{y}}{\Omega^{2} C^{2}}+\frac{U \Psi}{C^{2}}\right)\left(\frac{\Psi}{D}+\frac{\kappa U \Psi}{D \Omega C}-\frac{U U_{y} \Psi_{y}}{D \Omega^{2} C^{2}}\right) \mathrm{d} y+O(\varepsilon) \\
& =\frac{1}{2} \int_{g}^{h}\left[\left(\kappa+\frac{U \Omega}{C}\right) \frac{\omega \Psi^{2}}{D \Omega^{2} C^{2}}-\frac{\omega^{2} U_{y} \Psi \Psi_{y}}{D \Omega^{4} C^{4}}+\frac{U_{y}}{2 D C^{4}}\left(\frac{U^{2} \Psi_{y}^{2}}{\Omega^{4}}\right)\right] \mathrm{d} y+O(\varepsilon) \\
& =\frac{1}{2} \omega Q_{0}+O(\varepsilon) .
\end{aligned}
$$

It is clear that, for $\omega>0, Q_{0}$ must be chosen positive when the mode is right running, and negative when it is left running. Another conclusion we may draw is that, as far as the left-hand side of (4.13) describes the acoustic energy of the mode, it couples with the mean flow, to WKB accuracy, only where the specific shear $U_{y} / D$ changes in $X$.

The simplicity of (4.13) is elegant not only physically but also mathematically, because no $X$-derivatives of $\Psi$ and $\Psi_{y}$ are required. The existence of an adiabatic invariant is a remarkable result, and perhaps not entirely to be expected (Cooper \& Peake 2001; Peake \& Cooper 2001; Lloyd \& Peake 2013; Rienstra 2016b), because in general acoustic energy is not conserved in vortical flow. Although for slowly varying real, hard-wall modes in nearly parallel flow the time-averaged acoustic energy source $\langle\mathcal{D}\rangle(2.9 c)$ is $O(\varepsilon)$ :

$$
\langle\mathcal{D}\rangle=-\frac{1}{2} \operatorname{Re}\left[\mathrm{i} \frac{U U_{y y}}{D \Omega^{3} C^{3}} \Psi_{y}^{2}+O(\varepsilon)\right]=0+O(\varepsilon),
$$

the acoustic power is not necessarily conserved to $O(\varepsilon)$ because the volume integral of $\mathcal{D}$ over a distance $X=O(1)$ may be $O(\epsilon) O(1 / \epsilon)=O(1)$, finite.

This completes the leading-order solution of the slowly varying modes. From $\Psi_{0}$ the other amplitudes $A_{0}$ and $B_{0}$ follow with (4.6).

We end this analysis by checking the consistency of the resulting equation for $\Psi$ with the corresponding results for the potential flow problem, as considered for three dimensions in Rienstra $(1999,2003 a)$. Without shear, i.e. $U_{y}=0$, the mean flow is irrotational and can be given by a potential; $U$ and so $\Omega$ are then functions of $X$ only. If also the sound field is given by a potential $\Phi$ (no vortical perturbations like vorticity shed from sharp edges), such that

$$
A=-\mathrm{i} \kappa \Phi+\varepsilon \Phi_{X}, \quad B=\Phi_{y}, \quad \Psi=-\mathrm{i} D \Omega C \Phi-\varepsilon D\left(U \Phi_{X}+V \Phi_{y}\right)+O\left(\varepsilon^{2}\right),
$$

and boundary conditions

$$
\left.\begin{array}{l}
\Phi_{y}-\frac{D \Omega^{2} C^{2}}{\mathrm{i} \omega Z_{h}} \Phi=-\mathrm{i} \varepsilon h_{X} \kappa \Phi-\frac{\mathrm{i} \varepsilon}{\Phi}\left[\left(U \frac{\partial}{\partial X}+V \frac{\partial}{\partial y}\right)\left(\frac{D \Omega C \Phi^{2}}{\mathrm{i} \omega Z_{h}}\right)+(D U)_{X} \frac{\Omega C \Phi^{2}}{\mathrm{i} \omega Z_{h}}\right] \\
\Phi_{y}+\frac{D \Omega^{2} C^{2}}{\mathrm{i} \omega Z_{g}} \Phi=-\mathrm{i} \varepsilon g_{X} \kappa \Phi+\frac{\mathrm{i} \varepsilon}{\Phi}\left[\left(U \frac{\partial}{\partial X}+V \frac{\partial}{\partial y}\right)\left(\frac{D \Omega C \Phi^{2}}{\mathrm{i} \omega Z_{g}}\right)+(D U)_{X} \frac{\Omega C \Phi^{2}}{\mathrm{i} \omega Z_{g}}\right]
\end{array}\right\}
$$


we find upon substitution into (4.5)

$$
\Phi_{y y}+\left(\Omega^{2}-\kappa^{2}\right) \Phi=\frac{\mathrm{i} \varepsilon}{D \Phi}\left[\frac{\partial}{\partial X}\left(\left(\kappa+\frac{U \Omega}{C}\right) D \Phi^{2}\right)+\frac{\partial}{\partial y}\left(\frac{V \Omega}{C} D \Phi^{2}\right)\right]+O\left(\varepsilon^{2}\right) .
$$

After expanding $\Phi=\Phi_{0}+\varepsilon \Phi_{1}+O\left(\varepsilon^{2}\right)$, this leads (cf. Rienstra 1999) by the usual manipulations to the adiabatic invariant

$$
\begin{gathered}
\frac{\mathrm{d}}{\mathrm{d} X}\left[\left.\frac{D^{2} U \Omega C}{\mathrm{i} \omega Z_{h}} \Phi_{0}^{2}\right|_{h}+\left.\frac{D^{2} U \Omega C}{\mathrm{i} \omega Z_{g}} \Phi_{0}^{2}\right|_{g}+\int_{g}^{h}\left(\kappa+\frac{U \Omega}{C}\right) D \Phi_{0}^{2} \mathrm{~d} y\right] \\
=\frac{\mathrm{d}}{\mathrm{d} X}\left[\int_{g}^{h} \frac{D}{\Omega C}\left(\omega \kappa \Phi_{0}^{2}+U \Phi_{0 y}^{2}\right) \mathrm{d} y\right]=0,
\end{gathered}
$$

which agrees with $(4.13 a)$ and $(4.13 b)$, respectively, for $U_{y}=0$ if we identify $\Phi_{0}=$ $\mathrm{i} \Psi_{0} / D \Omega C$.

\section{Numerical evaluation of practical examples}

\subsection{Numerical solution of the Pridmore-Brown equation}

The heart of a numerical evaluation of the above solution is the solution of the Pridmore-Brown eigenvalue equation in $y((4.8 a)$ and $(4.8 b))$ along a grid of points in $x$. At each $x$, we consider eigenfunction $\Psi(X, y)$ as a function of $y$ only, and keep $X$ fixed.

A number of methods have been reported in the literature, e.g. Chebyshev spectral collocation methods acting on the vector of linearised Euler equations (Cooper \& Peake 2001; Lloyd \& Peake 2013), shooting methods (Vilenski \& Rienstra 2007b) and collocation methods (Oppeneer et al. 2011) acting directly on the Pridmore-Brown equation, each with their own benefits.

We will use here a method, published recently in Rienstra (2020), which is a classic Galerkin approach based on a symmetric bilinear form. We rewrite $(4.8 a)$ with boundary conditions $\mathrm{i} \omega \zeta_{h} \Psi^{\prime}(h)=\Omega^{2} \Psi^{\prime}(h)$ and $\mathrm{i} \omega \zeta_{g} \Psi^{\prime}(g)=-\Omega^{2} \Psi(g)$ in weak form, which means that we search for eigenfunction $\Psi$ and eigenvalue $\kappa$ such that for every test function $w$,

$$
\left.\frac{\Psi w}{\mathrm{i} \omega \zeta_{h}}\right|_{h}+\left.\frac{\Psi w}{\mathrm{i} \omega \zeta_{g}}\right|_{g}+\int_{g}^{h}-\frac{1}{\Omega^{2}} \Psi^{\prime} w^{\prime}+\left(1-\frac{\kappa^{2}}{\Omega^{2}}\right) \Psi w \mathrm{~d} y=0
$$

The prime indicates a partial derivative with respect to $y$. We assume that $\Omega \neq 0$ for any $y$ along the integration interval, to avoid the relatively unimportant critical layer solutions, not studied here; see Brambley et al. (2012). Here, $\Psi$ will be suitably normalised and then multiplied by a slowly varying amplitude $N$, determined as indicated above by (4.16) or (4.17). 
If we assume that $\Psi$ can be written as a sum over a function basis $\left\{\phi_{n}\right\}$ of Chebyshev polynomials,

$$
\Psi=\sum_{\mu=0}^{\infty} a_{\mu} \phi_{\mu}
$$

and we use the same basis for the test functions, i.e. $w=\phi_{v}, v=0,1, \ldots$, then $(5.1)$ becomes equivalent with the matrix equation

$$
\boldsymbol{M}(\kappa) \boldsymbol{a}=0, \quad \boldsymbol{a}=\left(a_{0}, a_{1}, \ldots\right)^{\mathrm{T}}
$$

for the doubly infinite symmetric matrix $\boldsymbol{M}$ with elements

$$
M_{\mu \nu}(\kappa)=\left.\frac{\phi_{\mu} \phi_{v}}{\mathrm{i} \omega \zeta_{h}}\right|_{h}+\left.\frac{\phi_{\mu} \phi_{v}}{\mathrm{i} \omega \zeta_{g}}\right|_{g}+\int_{g}^{h}\left\{-\frac{1}{\Omega^{2}} \phi_{\mu}^{\prime} \phi_{\nu}^{\prime}+\left(1-\frac{\kappa^{2}}{\Omega^{2}}\right) \phi_{\mu} \phi_{\nu}\right\} \mathrm{d} y .
$$

The $\kappa$-derivative $\boldsymbol{M}^{\prime}$ can be expressed in a similar way. The integrals of $\boldsymbol{M}$ and $\boldsymbol{M}^{\prime}$ are evaluated by the Gauss-Legendre method, which is efficient and needs relatively few points for a high accuracy.

The eigenvalue $\kappa$ is determined by a quadratically converging Newton-like iteration. Along the slowly varying duct, each previous $X$-position provides an excellent starting value, making, in general, no more than one iteration necessary. Since we deal with one mode, it is no loss of efficiency to find one eigenvalue at a time.

By using the same Gauss-Legendre points for integral (4.13) as for the elements of $\boldsymbol{M}$ and $\boldsymbol{M}^{\prime}$, we can normalise the found solutions as required. Here, we will take the transmitted power at the left entrance of the duct equal to $\mathcal{P}=1$ for right-running modes and at the right entrance equal to $\mathcal{P}=-1$ for left-running modes. Note that the normalisation is on $\Psi^{2}$, rather than $\Psi$, and we have to select a $\operatorname{sign}$ for $\Psi$ at each $x$-position. The correct sign is of course such that $\Psi$ is continuous in $x$.

The integrals over $\kappa$ and $G / F$ may be constructed by incremental trapezoidal integration.

\subsection{A practical example}

A typical and relevant example may be a linearly sheared mean flow. Although our results are valid for any mean flow profile (provided the slowly varying mean flow satisfies (3.8)), linear shear is most interesting in two respects. First, the adiabatic invariant is 'complete', i.e. really a constant of the problem, and second, the mean flow may be given explicitly, without an integration along the streamlines.

Although a linear profile of a flow with friction is eventually not realistic (a parabolic profile for laminar flow or 1/7-power profiles for turbulent flow is more likely; Hinze 1972), it is not entirely unphysical. For example, a realisation of the model may be found in the annular duct behind the fan of a modern, high-bypass turbofan engine, which leads a small fraction of the flow into the core of the engine. Since this annular duct is narrow compared to the diameter, a 2-D approximation is valid for axially propagating modes. Moreover, its mean flow is the continuation of the strongly sheared boundary layer along the inner hub of the engine's inlet duct, thus making a linearly sheared profile relevant. 

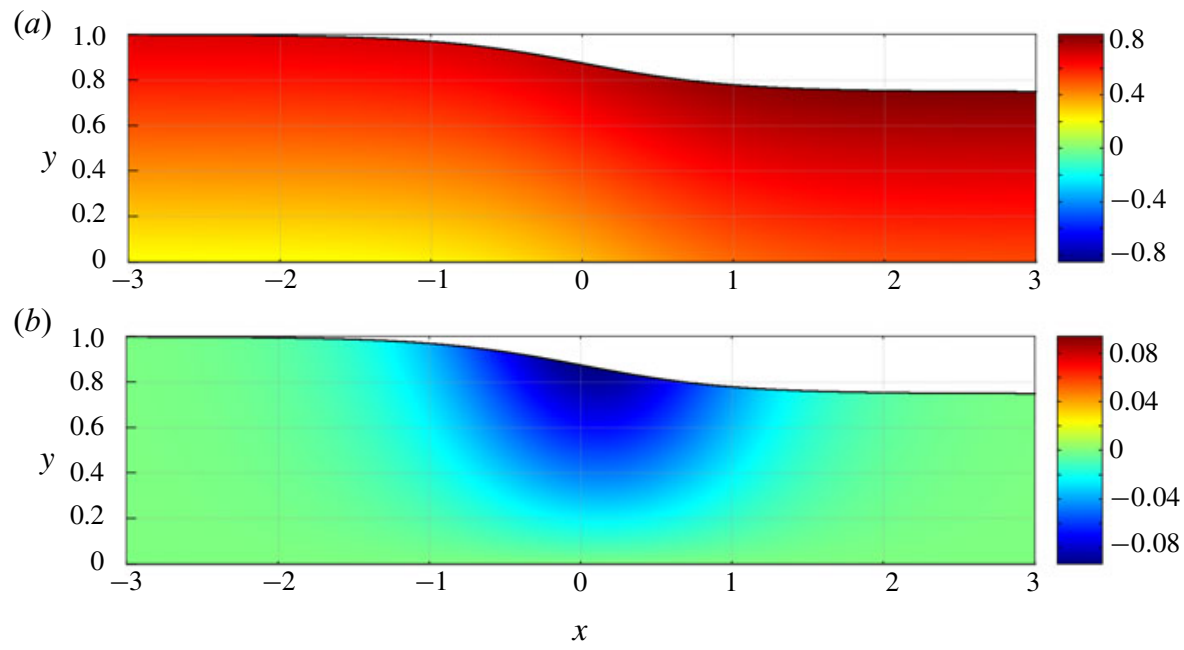

Figure 2. Mean flow in $x, y$-domain. (a) Mean flow $U$. (b) Mean flow $V$.

The configuration chosen is as follows. The right-running mean flow is described by the problem parameters

$$
\lambda=0.5, \quad \gamma=1.4, \quad D_{\text {in }}=1, \quad \tau_{\text {in }}=0.2, \quad \mathcal{F}=0.4496, \quad E=2.52, \quad(5.5 a-f)
$$

and the duct geometry (with inlet at $x=-3$ ) by

$$
h(x)=1-\frac{1}{8}(1+\tanh x), \quad g(x)=0, \quad-3<x<3,
$$

giving an estimated $\varepsilon \simeq 0.1$. See figure 2 for the $U$ and $V$ components. The decreasing duct height results in an increase of $U$ with a slight decrease of $U_{y}=\sigma ; V$ is obviously at its maximum near $x=0$ at the upper wall.

For the acoustic part we consider a hard-wall and a soft-wall configuration, both with $\omega=15$. The hard-wall case has in total 10 cut-on modes: $p_{1}, \ldots, p_{5}$ right running and $p_{-1}, \ldots, p_{-5}$ left running. The modes $p_{6}$ and $p_{-6}$ start cut-on at $x=-3$ but become cut-off near $x=-0.055$, where $F(X)=0$, and the approximate solution breaks down. Since beyond this point the modal energy vanishes, mode $p_{6}$ has to reflect here into a multiple of $p_{-6}$. For this reason the point is called a turning point. In its neighbourhood a local analysis is required (Rienstra 2000; Cooper \& Peake 2001). Since we have not included this here, we exclude the modes $p_{6}$ and $p_{-6}$. For the soft-wall case with $Z_{h}=Z_{g}=1+2 \mathrm{i}$ we consider the right running $p_{2}$ and left running $p_{-2}$.

With matrix $\boldsymbol{M}$ chopped off to just $21 \times 21$ (21 Chebyshev terms) and for the integrals using only 40 Gauss-Legendre points we obtain all eigenvalues correct in 11 digits, as can be estimated by doubling these numbers a few times. We expect the same accuracy for the eigenfunctions. The number of $\kappa$-iterations, using the result of the previous $X$-position as starting value, is typically 1 (depending on the number of $X$-positions; here 2400). Per $X$ and 400 plot points in $y$, this takes altogether approximately $0.04 \mathrm{~s}$ (Matlab2018, Intel Core i5-4670 CPU@ $3.4 \mathrm{GHz}$ ).

We see that the effects of the sheared mean flow properties are various; see figures 3 and 4 with snapshots of the pressure distribution in the hard-wall duct. For the lowest modal orders, especially upstream running, the asymmetry of the flow is reflected in 
(a)

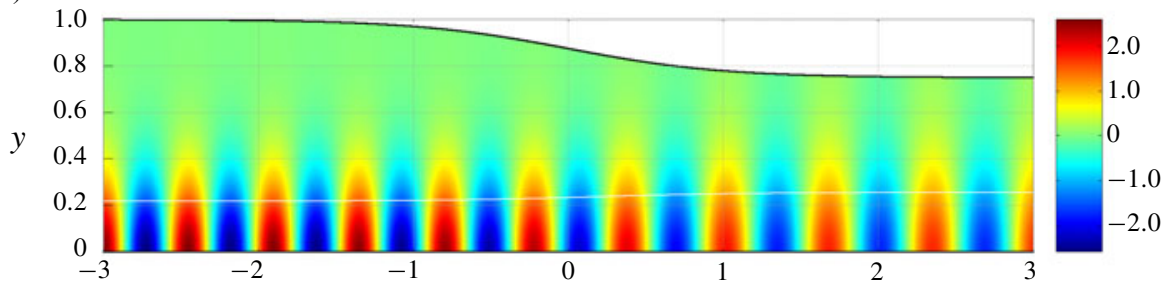

(b)
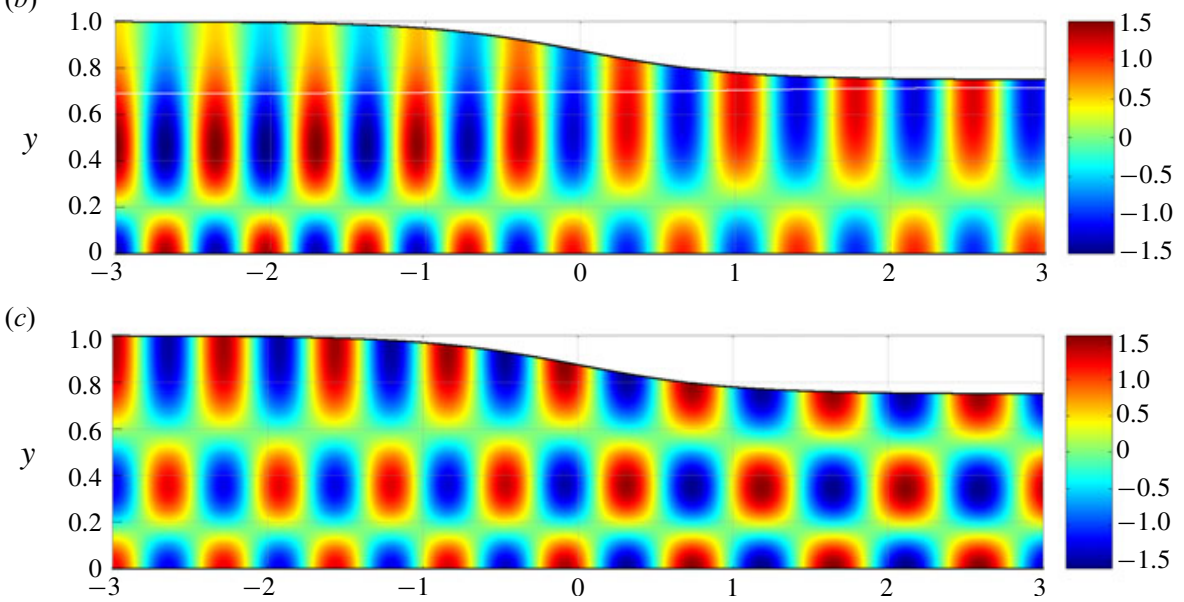

(d)

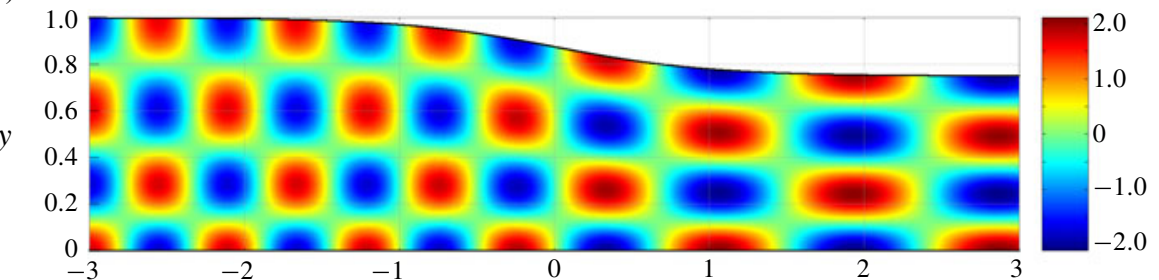

(e)

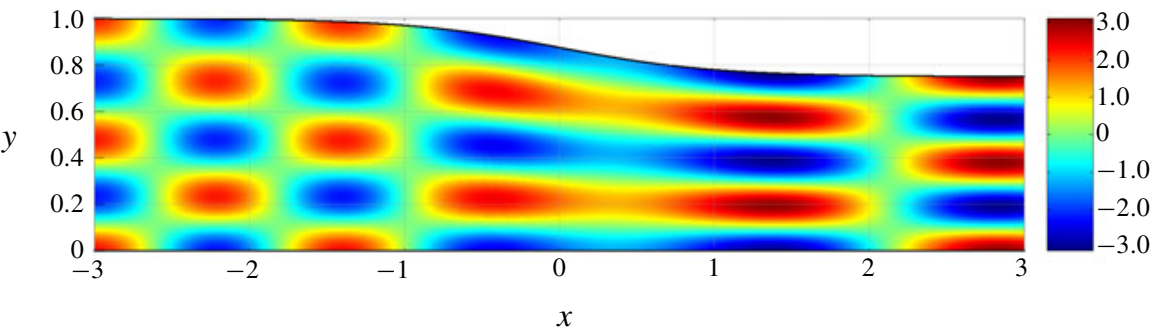

FIGURE 3. Pressure field snapshots (order $1, \ldots, 5)$ for $\omega=15$ and $\mathcal{P}=1$ (right running). (a) $\kappa_{1}=11.47, \ldots, 9.33 . \quad$ (b) $\kappa_{2}=9.71, \ldots, 8.27 . \quad$ (c) $\kappa_{3}=8.77, \ldots, 6.63 . \quad(d) \quad \kappa_{4}=$ 7.07, .., 3.34. (e) $\kappa_{5}=4.01, \ldots,-2.26$.

asymmetric mode shapes. The right-running modes exist only near the lower wall $y=0$ and are negligible at the other side. The upstream-running modes behave the same upside down. This behaviour is due to the sign of $\Omega^{2}-\kappa^{2}$ : for large enough $\omega$, the mode is oscillatory in $y$ when $\Omega^{2}-\kappa^{2}$ is positive, and exponentially decaying otherwise (Rienstra 2020). For the present, linear, mean flow profile the $y$-interval with oscillatory 
(a)
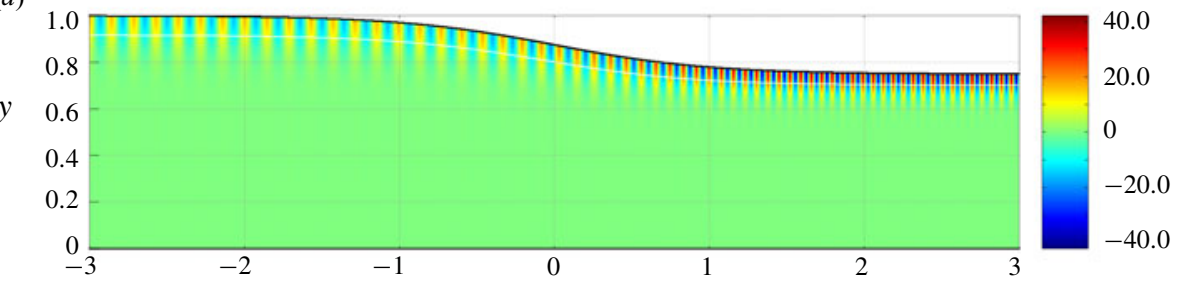

(b)

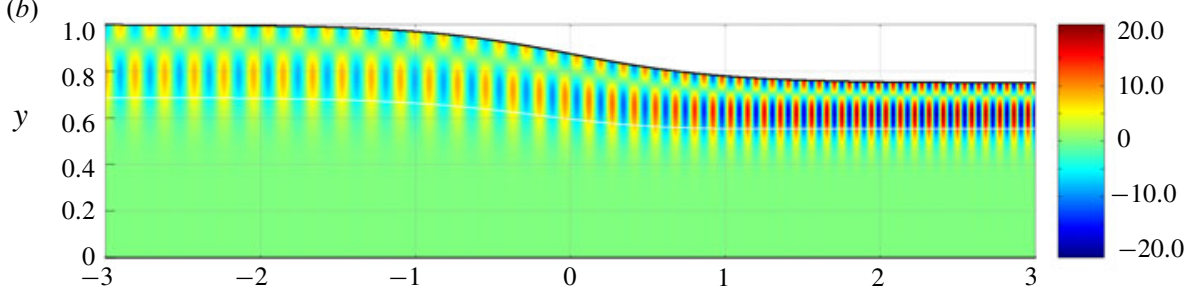

(c)

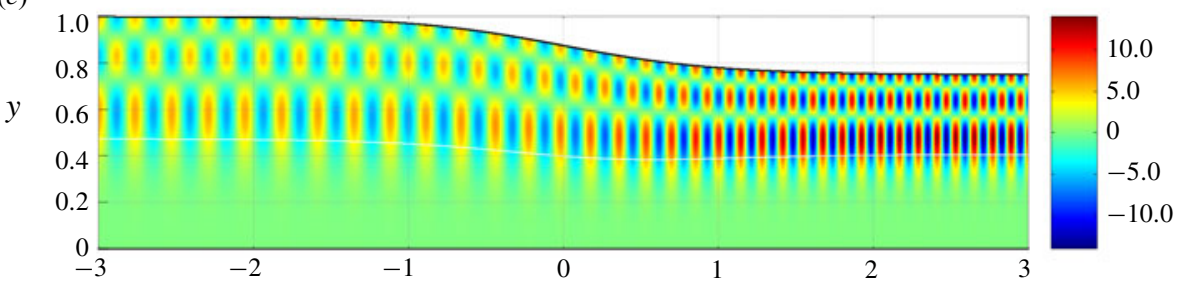

(d)

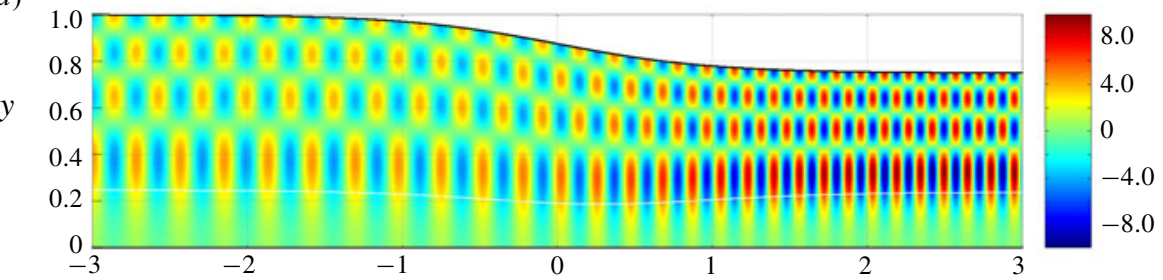

(e)

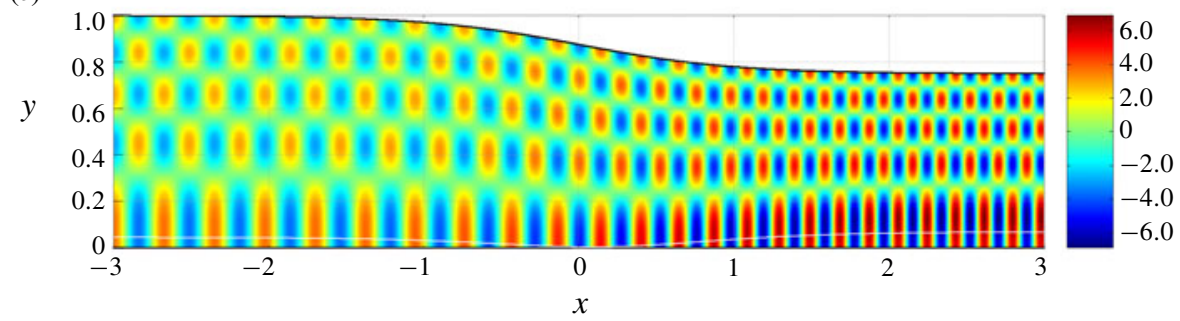

FIgURE 4. Pressure field snapshots (order $-1, \ldots,-5$ ) for $\omega=15$ and $\mathcal{P}=-1$ (left running). (a) $\kappa_{-1}=-43.78, \ldots,-100.04$. (b) $\kappa_{-2}=-32.82, \ldots,-69.40$. (c) $\kappa_{-3}=$ $-26.55, \ldots,-52.84$. (d) $\kappa_{-4}=-22.13, \ldots,-41.69$. (e) $\kappa_{-5}=-19.27, \ldots,-34.44$.

behaviour is either the full interval, or a part adjacent to the upper or lower duct wall. The contours corresponding to $\Omega^{2}-\kappa^{2}=0$ are indicated in the figures by white lines.

In figures 5 and 6 the mode shapes in $y$ for various $x$ are given in detail. Indeed modes $p_{1}, p_{-1}, p_{-2}, p_{-3}$, are practically vanishing at the upper, respectively lower, wall. As a 
(a)

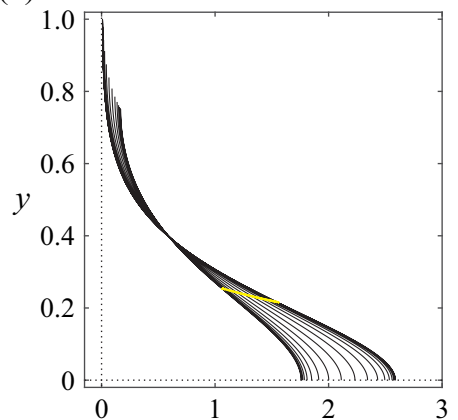

(b)

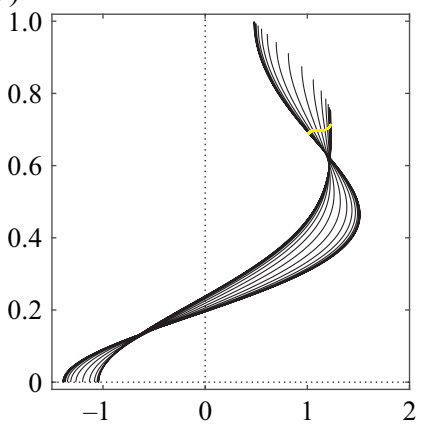

(c)

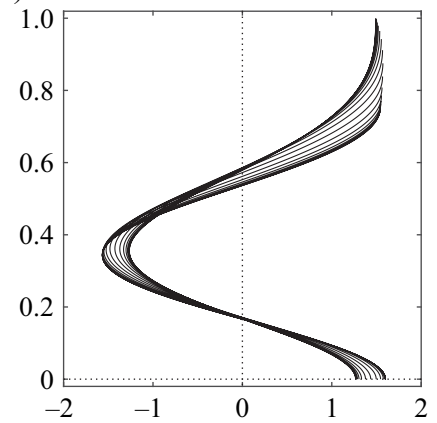

(d)

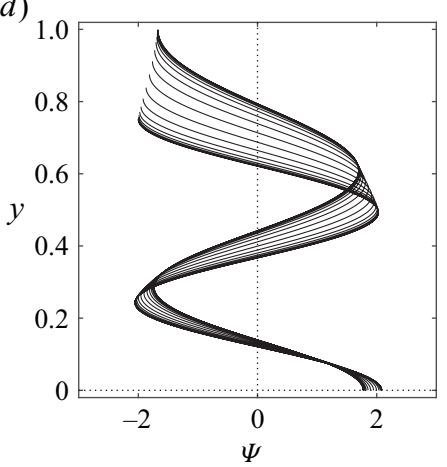

(e)

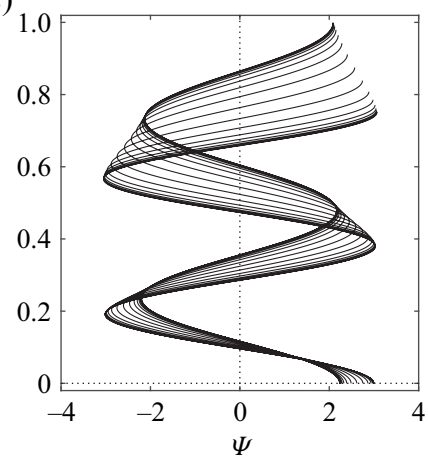

FIGURE 5. Right-running $n$ th-order modal functions $\Psi_{n}(X, y)$ for $\omega=15$, as a function of $y-g$ for varying $X$. $\Psi_{6}$ becomes cut-off and is not included. (a) $\Psi_{1}$. (b) $\Psi_{2}$. (c) $\Psi_{3}$. (d) $\Psi_{4}$. (e) $\Psi_{5}$.

result, these modes will not change if the respective wall is lined. Any attenuation due to lining will have to come from the opposite wall. The contours of $\Omega^{2}-\kappa^{2}=0$ in the mode shapes figures are given by yellow lines.

The modal wavenumbers $\kappa_{n}$ as a function of $x$ are given in figure 7 . The analogue of the Doppler effect in uniform flow, of increasing wavenumbers for upstream-running modes and decreasing wavenumbers on downstream-running modes, is clearly present.

Near $x=0.4$, the value of $\kappa_{5}$ changes sign, the physical relevance of which is visible in figure 3(e), where the modal wavelength suddenly becomes large, and (this is clearer in an animation) the phase velocity changes from positive to negative. The mode seems to propagate to the right for $x<0.4$ and to the left for $x>0.4$. This, however, is an optical illusion. The energy of the mode remains right-running everywhere.

As the selected impedance does not have much attenuation, the corresponding figures for the lined wall case are comparable. The snap shots of the pressure distribution, figure 8 , show the expected Doppler compression upstream and stretching downstream, and the decay to the right for the right-running mode $p_{2}$ and to the left for the left-running mode $p_{-2}$. We see here and in figure 9 again that the field of $p_{-2}$ practically vanishes near the lower wall.

The attenuation is better displayed by the transmitted power $10 \log _{10}|\mathcal{P}|$ in $\mathrm{dB}$, shown in figure 10. For comparison, the contributions of the exponential, given by $20 \log _{10}(\mathrm{e}) \operatorname{Im} \int^{x} \kappa_{ \pm 2} \mathrm{~d} x^{\prime}$, has been added as dashed lines. The difference between the exponential and the power is very small (on the logarithmic scale), and the role of the 

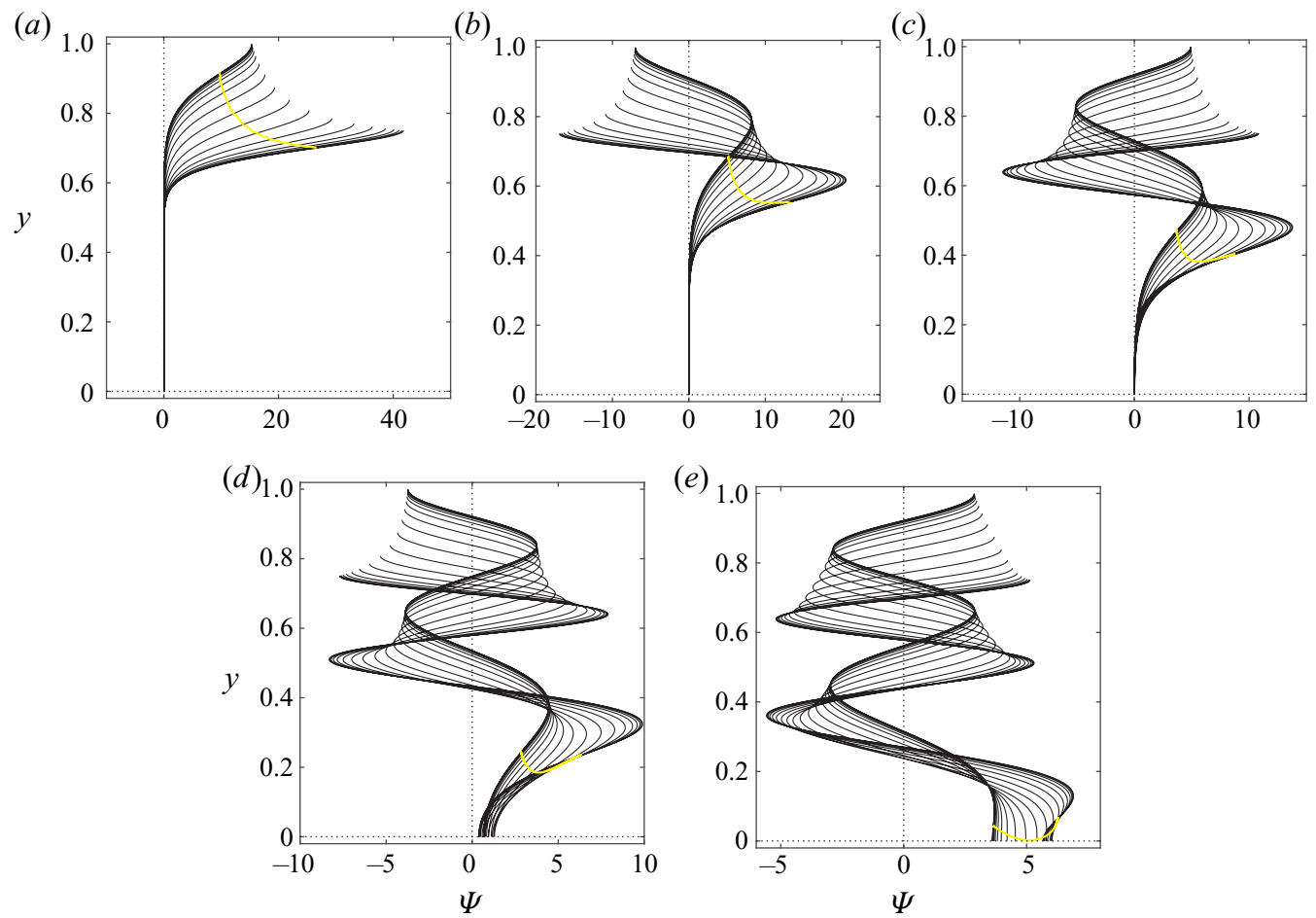

FIGURE 6. Left-running $n$ th-order modal functions $\Psi_{n}(X, y)$ for $\omega=15$, as a function of $y-g$ for varying $X . \Psi_{-6}$ becomes cut-off and is not included. (a) $\Psi_{-1}$. (b) $\Psi_{-2}$. (c) $\Psi_{-3}$. (d) $\Psi_{-4}$. (e) $\Psi_{-5}$.

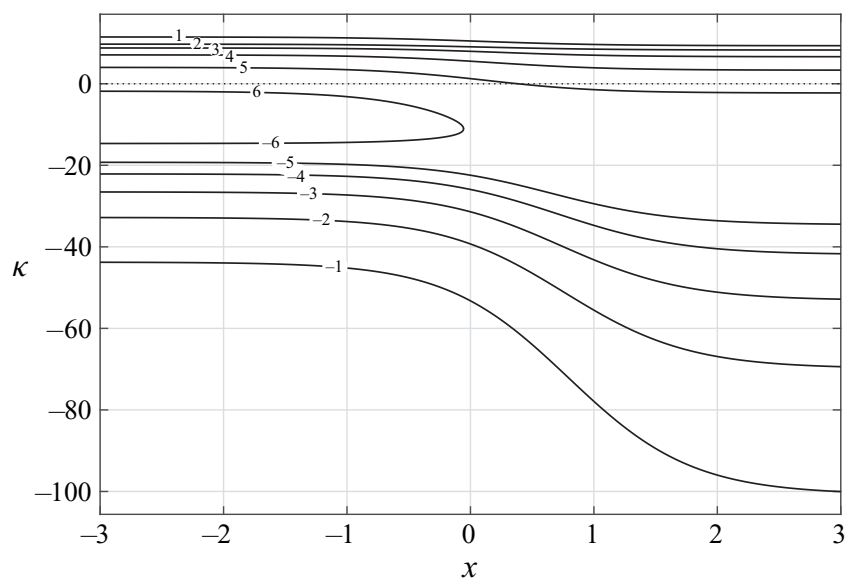

FIgURE 7. Axial wavenumbers $\kappa_{1}, \ldots, \kappa_{-1}$ (from top to bottom) as a function of $x$. $\kappa_{6}$ and $\kappa_{-6}$ become cut-off and merge together at $x=-0.055$. 
(a)

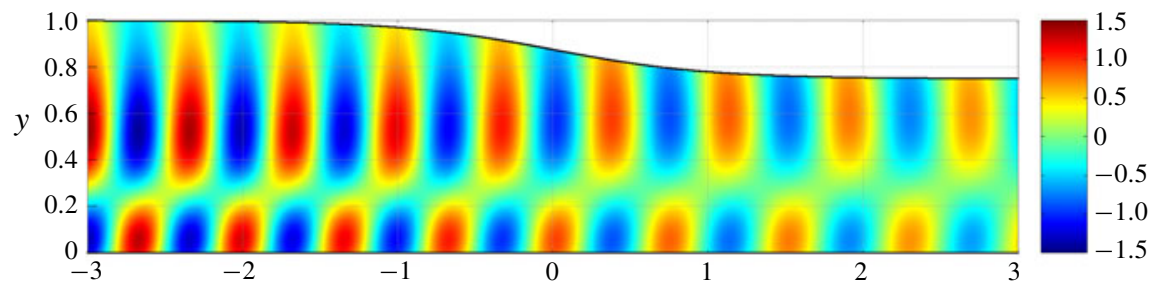

(b)

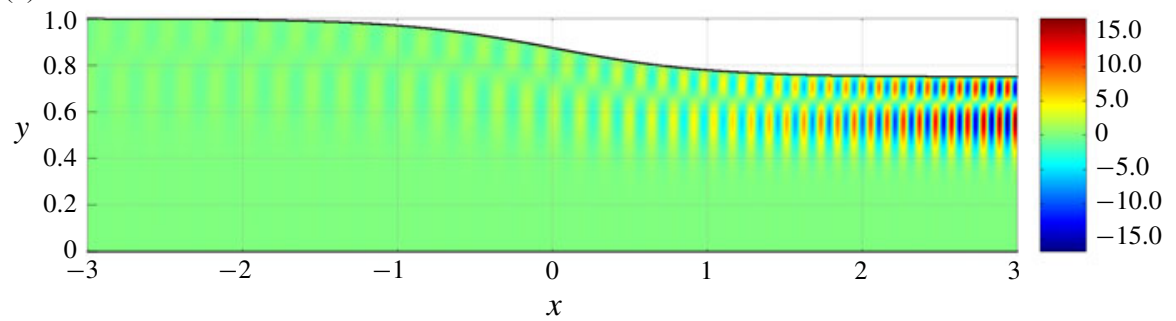

FIgURE 8. Pressure field snapshots (orders \pm 2 ) for $\omega=15$ and $Z=1+2 \mathrm{i}$.

(a) $\kappa_{2}=9.56-0.84 \mathrm{i}, \ldots, 7.99-0.13 \mathrm{i}$. (b) $\kappa_{-2}=-30.25+0.38 \mathrm{i}, \ldots,-61.22+0.53 \mathrm{i}$.

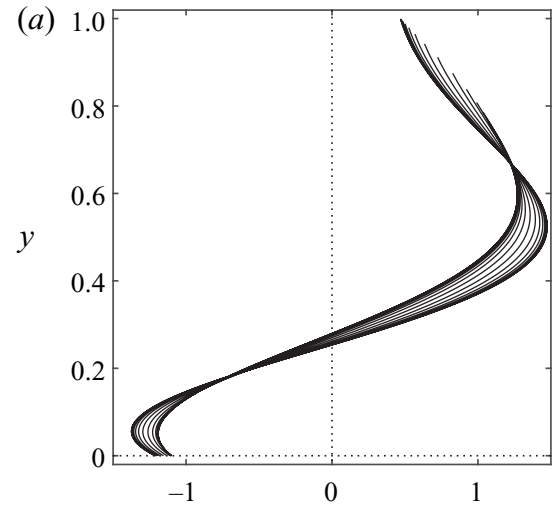

(b)

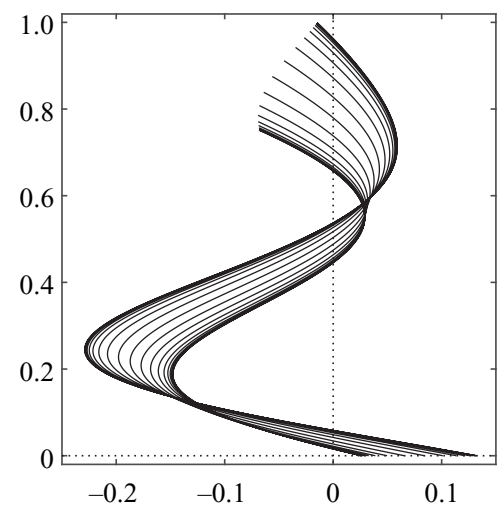

(c)
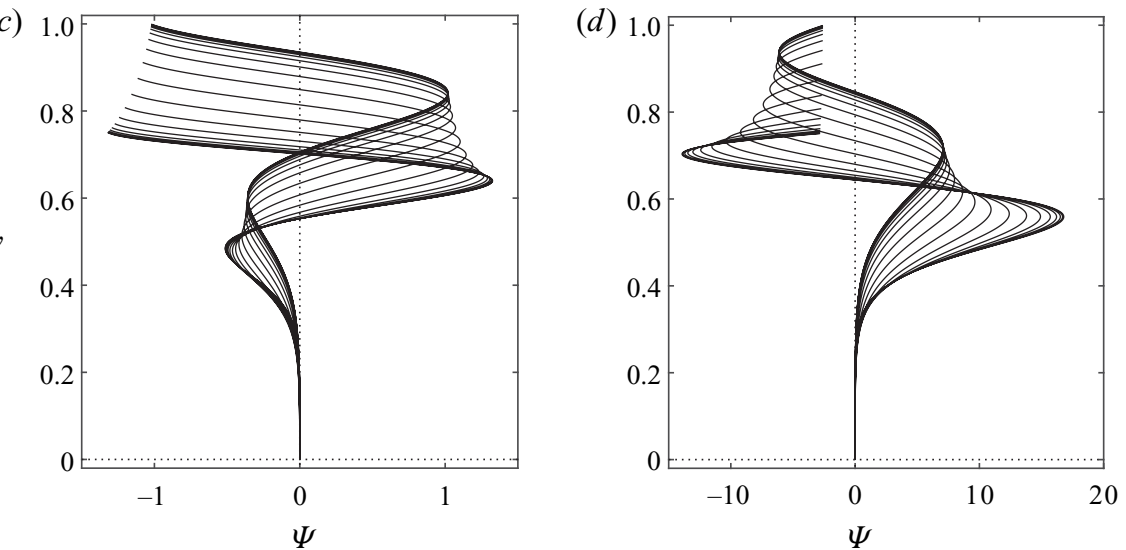

FIGURE 9. Real and imaginary parts of modal functions $\Psi_{ \pm 2}(X, y)$ for $\omega=15, Z=1+2 \mathrm{i}$, as a function of $y-g$ for varying $X .(a) \operatorname{Re}\left(\Psi_{2}\right)$. (b) $\operatorname{Im}\left(\Psi_{2}\right)$. (c) $\operatorname{Re}\left(\Psi_{-2}\right)$. (d) $\operatorname{Im}\left(\Psi_{-2}\right)$. 


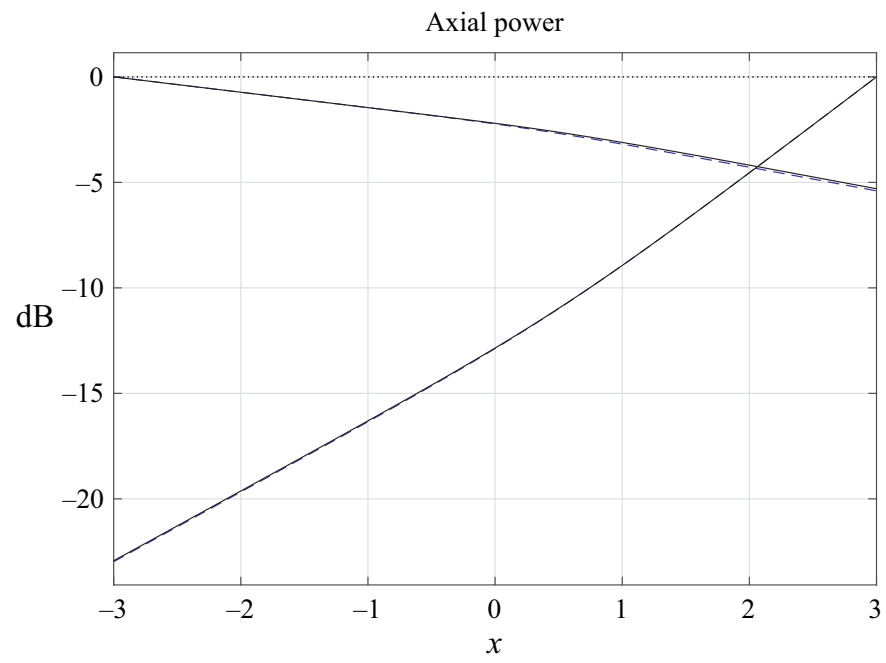

FIGURE 10. Axial acoustic power $10 \log _{10}|\mathcal{P}|$ in $\mathrm{dB}$ of right-running mode 2 and left-running mode -2 as a function of $x$.

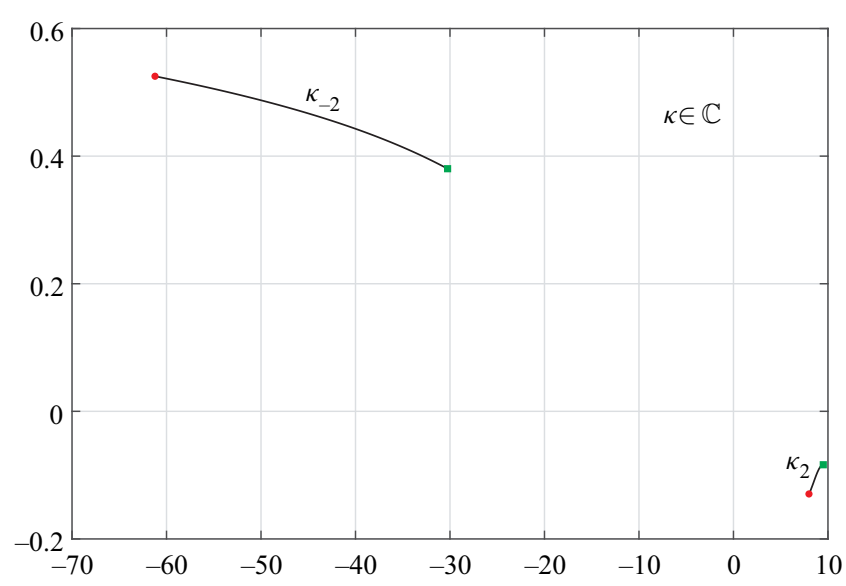

FIGURE 11. Complex axial wavenumbers $\kappa_{ \pm 2}$ varying from $x=-3$ ( $\boldsymbol{\square}$, green) to $x=3$ $(\bullet$, red).

modal shape function in the decay is small here. The lines are not straight because $\kappa_{ \pm 2}$ vary with $x$; see figure 11 .

\section{Conclusions}

The theory of slowly varying modes of WKB type in lined ducts with mean flow is fairly well established for irrotational (potential) mean flow, because the slowly varying mean flow equations can be solved practically analytically and the modal amplitude equations can be integrated completely to a conserved quantity called an adiabatic invariant. This is not generally the case for rotational mean flows. The equations for the mean flow as well as the modal amplitudes require numerical integration, which is a practical 
inconvenience, but more importantly, as far as we can tell, no simple adiabatic invariant is available.

Since conserved quantities (even approximate) have a physical meaning that may uncover deeper relations, it is of interest to study their occurrence and possible configurations allowing for their existence. This was a main goal of the present study.

In the present type of slowly varying modal wave forms, an adiabatic invariant takes the form of a vanishing (axial) $X$-derivative of a cross-sectional integral of a combination of squares and products of the modal shape function $\Psi$ and its cross-wise derivatives $\Psi_{y}, \Psi_{z}$.

In standard WKB analysis it is always possible to construct a vanishing cross-sectional integral of products between $\Psi, \Psi_{y}, \Psi_{z}$ and $\Psi_{X}$. Only rarely can the integral be split up in a part with and a part without $\Psi_{X}$, such that the $X$-derivatives are rearranged and taken outside the integral. If this is the case, the obtained expression may be called an 'incomplete adiabatic invariant' if it is still $X$ dependent, or a 'complete adiabatic invariant' (or just 'adiabatic invariant') if it is a constant.

Although it is still an open question under what conditions invariants exist in general (for example, in 3-D cylindrical configurations with shear flow), the present study showed that the simplifications due to a 2-D duct provide conditions that allow remarkable progress in several ways. For a general sheared mean flow, the mean flow and amplitude equations cannot be solved without a numerical integration but the amplitudes satisfy an equation that is almost an adiabatic invariant. If the specific mean vorticity remains constant in the axial direction (rather than constant along streamlines, as it does in general), the incomplete adiabatic invariant reduces to a complete one.

This is just what happens for the particular case of a linearly sheared mean flow. Furthermore, not only is the specific mean vorticity constant, but the whole flow field can be determined practically analytically (up to an algebraic equation).

In conclusion, there is a remarkable, but apparently uncorrelated coincidence for 2-D modes in slowly varying linearly sheared flow: (i) the governing Pridmore-Brown equation can be solved analytically; (ii) the mean flow equations can be solved (practically) analytically; (iii) the modal amplitude equation can be integrated completely.

Unfortunately, the analytical solution (Goldstein \& Rice 1973) of the Pridmore-Brown equation, being the difference of exponentially large functions, did not prove to be very useful here. The simplicity of the other two results, however, makes the linear shear flow problem about as simple as the potential flow problem.

Finally we note that, apart from the existence problem of adiabatic invariants in general, a problem that could be subject of future study is the cut-on-cut-off transition (or turning point problem) of the present slowly varying modes.

\section{Declaration of interests}

The author reports no conflict of interest.

\section{Appendix A}

A useful relation for sufficiently smooth function $\mathcal{G}$ and solutions $\Psi, \kappa$ of the Pridmore-Brown eigenvalue equation $(4.8 a)$ is the integral

$$
\int_{g}^{h} \Psi^{2} \mathcal{G} \mathrm{d} y=\int_{g}^{h}\left[\frac{\mathcal{G}}{\Omega^{2}}\left(\kappa^{2} \Psi^{2}+\Psi_{y}^{2}\right)+\frac{\mathcal{G}_{y}}{\Omega^{2}} \Psi \Psi_{y}\right] \mathrm{d} y-\left[\frac{\mathcal{G} \Psi \Psi_{y}}{\Omega^{2}}\right]_{g}^{h}
$$


We prove it by noting that from the defining equation we have

$$
\Psi^{2} \mathcal{G}=\frac{\kappa^{2}}{\Omega^{2}} \Psi^{2} \mathcal{G}-\left(\frac{\Psi_{y}}{\Omega^{2}}\right)_{y} \Psi \mathcal{G},
$$

and use partial integration. In particular, with $\mathcal{G}=U /\left(D \Omega C^{3}\right), \mathcal{G}_{y}=\omega U_{y} /\left(D \Omega^{2} C^{4}\right)$

$$
\begin{aligned}
\int_{g}^{h} & \left(\kappa+\frac{U \Omega}{C}\right) \frac{\Psi^{2}}{D \Omega^{2} C^{2}}-\frac{\omega U_{y}}{D \Omega^{4} C^{4}} \Psi \Psi_{y} \mathrm{~d} y \\
= & \int_{g}^{h} \frac{\kappa}{D \Omega^{2} C^{2}} \Psi^{2} \mathrm{~d} y+\int_{g}^{h} \frac{U}{D \Omega C^{3}} \Psi^{2}-\frac{\omega U_{y}}{D \Omega^{4} C^{4}} \Psi \Psi_{y} \mathrm{~d} y \\
= & \int_{g}^{h} \frac{1}{D \Omega^{3} C^{3}}\left(\omega \kappa \Psi^{2}+U \Psi_{y}^{2}\right) \mathrm{d} y-\left[\frac{U}{D \Omega^{3} C^{3}} \Psi \Psi_{y}\right]_{g}^{h} .
\end{aligned}
$$

\section{REFERENCES}

Batchelor, G. K. 1967 An Introduction to Fluid Mechanics. Cambridge University Press.

Bouthier, M. 1972 Stabilité linéaire des écoulements presque parallèles. J. Méc. 11 (4), 599-621.

BRAmbley, E. J. \& PEAKe, N. 2008 Sound transmission in strongly curved slowly varying cylindrical ducts with flow. J. Fluid Mech. 596, 387-412.

BRAmbley, E. J., RienstRa, S. W. \& DARAU, M. 2012 The critical layer in linear-shear boundary layers over acoustic linings. J. Fluid Mech. 710, 545-568.

Cooper, A. J. \& Peake, N. 2001 Propagation of unsteady disturbances in a slowly varying duct with mean swirling flow. J. Fluid Mech. 445, 207-234.

GARRETT, C. J. R. 1967 Discussion: the adiabatic invariant for wave propagation in a nonuniform moving medium. Proc. R. Soc. A 299 (1456), 26-27.

Goldstein, M. \& Rice, E. 1973 Effect of shear on duct wall impedance. J. Sound Vib. 30 (1), 79-84.

HINZE, J. O. 1972 Turbulence, 2nd edn. McGraw-Hill.

INGARD, K. U. 1959 Influence of fluid motion past a plane boundary on sound reflection, absorption, and transmission. J. Acoust. Soc. Am. 31 (7), 1035-1036.

Lloyd, A. E. D. \& PEAKE, N. 2013 The propagation of acoustic waves in a slowly varying duct with radially sheared axial mean flow. J. Sound Vib. 332, 3937-3946.

MYERS, M. K. 1980 On the acoustic boundary condition in the presence of flow. J. Sound Vib. 71 (3), 429-434.

MYERs, M. K. 1991 Transport of energy by disturbances in arbitrary steady flows. J. Fluid Mech. 226, $383-400$.

Nayfeh, A. H., Kaiser, J. E. \& Telionis, D. P. 1975 Transmission of sound through annular ducts of varying cross sections. AIAA J. 13 (1), 60-65.

NAYfeh, A. H. \& Telionis, D. P. 1973 Acoustic propagation in ducts with varying cross sections. J. Acoust. Soc. Am. 54 (6), 1654-1661.

Oppeneer, M., Rienstra, S. W., Lazeroms, W., Mattheij, R. M. M. \& Sijtsma, P. 2011 Acoustic modes in a duct with slowly varying impedance and non-uniform mean flow and temperature. AIAA Paper 2011-2871.

Oppeneer, M., Rienstra, S. W. \& SiJTsma, P. 2016 Efficient mode-matching based on closed form integrals of Pridmore-Brown modes. AIAA J. 54 (1), 266-279.

OVEnden, N. C. 2002 Near cut-on/cut-off transitions in lined ducts with flow. AIAA Paper 2002-2445.

OvENDEN, N. C. 2005 A uniformly valid multiple scales solution for cut-on cut-off transition of sound in flow ducts. J. Sound Vib. 286, 403-416.

Ovenden, N. C., Eversman, W. \& Rienstra, S. W. 2004 Cut-on cut-off transition in flow ducts: comparing multiple-scales and finite-element solutions. AIAA Paper 2004-2945.

Ovenden, N. C. \& Rienstra, S. W. 2004 Mode-matching strategies in slowly varying engine ducts. AIAA J. 42 (9), 1832-1840. 
PEAKe, N. \& COOPER, A. J. 2001 Acoustic propagation in ducts with slowly varying elliptic cross-section. J. Sound Vib. 243 (3), 381-401.

PRIDMORE-BROWN, D. 1958 Sound propagation in a fluid flowing through an attenuating duct. J. Fluid Mech. 4 (4), 393-406.

RIENSTRA, S. W. 1999 Sound transmission in slowly varying circular and annular ducts with flow. J. Fluid Mech. 380, 279-296.

Rienstra, S. W. 2000 Cut-on, cut-off transition of sound in slowly varying flow ducts. Aerotec. Missili Spazio 79 (3-4), 93-96.

RiEnstRA, S. W. 2003a Sound propagation in slowly varying lined flow ducts of arbitrary cross section. J. Fluid Mech. 495, 157-173.

Rienstra, S. W. 2003 b A classification of duct modes based on surface waves. Wave Motion 37, 119-135.

Rienstra, S. W. $2016 a$ Fundamentals of duct acoustics. In Progress in Simulation, Control and Reduction of Ventilation Noise, 16-18 November 2015, Von Karman Institute Lecture Series 2016-02 (ed. C. Schram). Von Karman Institute for Fluid Dynamics.

Rienstra, S. W. $2016 b$ Sound propagation in slowly varying 2D duct with shear flow. AIAA Paper 2016-2925.

Rienstra, S. W. 2020 Numerical and asymptotic solutions of the Pridmore-Brown equation. AIAA J. 58 (7), 3001-3018.

Rienstra, S. W. \& Hirschberg, A. 2004 An Introduction to Acoustics. Technische Universiteit Eindhoven. Extended and revised edition of IWDE 92-06. Available at: http://www.win.tue.nl/ $\sim$ sjoerdr/papers/boek.pdf.

VILENSKI, G. G. \& RIENSTRA, S. W. $2007 a$ On hydrodynamic and acoustic modes in a ducted shear flow with wall lining. J. Fluid Mech. 583, 45-70.

Vilenski, G. G. \& Rienstra, S. W. $2007 b$ Numerical study of acoustic modes in ducted shear flow. J. Sound Vib. 307 (3-5), 610-626. 